\title{
ITERATIONS OF HOLOMORPHIC COLLET-ECKMANN MAPS: CONFORMAL AND INVARIANT MEASURES. APPENDIX: ON NON-RENORMALIZABLE QUADRATIC POLYNOMIALS
}

\author{
FELIKS PRZYTYCKI
}

\begin{abstract}
We prove that for every rational map on the Riemann sphere $f: \overline{\mathbb{C}} \rightarrow \overline{\mathbb{C}}$, if for every $f$-critical point $c \in J$ whose forward trajectory does not contain any other critical point, the growth of $\left|\left(f^{n}\right)^{\prime}(f(c))\right|$ is at least of order $\exp Q \sqrt{n}$ for an appropriate constant $Q$ as $n \rightarrow \infty$, then $\operatorname{HD}_{\text {ess }}(J)=$ $\alpha_{0}=\operatorname{HD}(J)$.

Here $\operatorname{HD}_{\text {ess }}(J)$ is the so-called essential, dynamical or hyperbolic dimension, $\operatorname{HD}(J)$ is Hausdorff dimension of $J$ and $\alpha_{0}$ is the minimal exponent for conformal measures on $J$.

If it is assumed additionally that there are no periodic parabolic points then the Minkowski dimension (other names: box dimension, limit capacity) of $J$ also coincides with $\operatorname{HD}(J)$.

We prove ergodicity of every $\alpha$-conformal measure on $J$ assuming $f$ has one critical point $c \in J$, no parabolic, and $\sum_{n=0}^{\infty}\left|\left(f^{n}\right)^{\prime}(f(c))\right|^{-1}<\infty$.

Finally for every $\alpha$-conformal measure $\mu$ on $J$ (satisfying an additional assumption), assuming an exponential growth of $\left|\left(f^{n}\right)^{\prime}(f(c))\right|$, we prove the existence of a probability absolutely continuous with respect to $\mu, f$-invariant measure.

In the Appendix we prove $\operatorname{HD}_{\text {ess }}(J)=\operatorname{HD}(J)$ also for every non-renormalizable quadratic polynomial $z \mapsto z^{2}+c$ with $c$ not in the main cardioid in the Mandelbrot set.
\end{abstract}

\section{INTRODUCTION}

Let $f: \overline{\mathbb{C}} \rightarrow \overline{\mathbb{C}}$ be a rational mapping of the Riemann sphere $\overline{\mathbb{C}}$. Let $\operatorname{HD}_{\text {ess }}(J)$ denote the essential Hausdorff dimension of the Julia set $J=J(f)$. It is defined by $\mathrm{HD}_{\text {ess }}(J)=\sup \{\mathrm{HD}(\mu): \mu$ is a probability, $f$-invariant measure on $J, \chi(\mu)>0\}$.

Here $\operatorname{HD}(\mu)$ denotes the Hausdorff dimension of the measure $\mu$, i.e. infimum of the Hausdorff dimensions of sets of full measure $\mu$.

The number $\chi(\mu)=\int \log \left|f^{\prime}\right| d \mu$ denotes the Lyapunov exponent. The derivative $\left|f^{\prime}\right|$ is considered in the spherical Riemann metric on $\overline{\mathbb{C}}$.

One calls a map $f^{m}: Y \rightarrow J$ a horseshoe (by analogy with a 2-dimensional Smale's horseshoe) if there exist pairwise disjoint compact sets $Y_{1}, \ldots, Y_{k} \subset Y$ such

Received by the editors January 18, 1995 and, in revised form, July 28, 1995 and June 13, 1996.

1991 Mathematics Subject Classification. Primary 58F23.

The author acknowledges support by Polish KBN Grants 210469101 and 2 P301 01307 "Iteracje i Fraktale". He expresses also his gratitude to the Universities at Orleans and at Dijon in France, where parts of this paper were written. 
that $\left.f^{m}\right|_{Y_{j}}$ is an injection onto $Y$ and $\left|\left(f^{t m}\right)^{\prime}\right|>1$ for $t$ large enough, for each $j=$ $1, \ldots, k$. It is easy to see that the set of points which never leave $Y$ under iteration of $f^{m}$ is an expanding isolated Cantor set. Expanding means that $\left|\left(f^{t m}\right)^{\prime}\right|>1$ on this set for $t$ large enough. A set $X$ is called isolated if there is an open set $U \supset X$ such that there is no forward $f$-invariant $X^{\prime} \subset U$ strictly bigger than $X$.

One can prove by A. Katok's methods $[K]$ the existence and abundance of horseshoes. More precisely one can prove

$$
\begin{array}{r}
\operatorname{HD}_{\text {ess }}(J)=\sup \{\operatorname{HD}(X): X \subset J, X \text { being an } f \text {-invariant } \\
\quad \text { isolated expanding Cantor set }\},
\end{array}
$$

see [PUbook, Ch. "Conformal measures"] for details.

A probability measure $\mu$ on $J$ is called $\alpha$-conformal if for every Borel $B \subset J$ on which $f$ is injective $\mu(f(B))=\int_{B}\left|f^{\prime}\right|^{\alpha} d \mu$. In particular $\left|f^{\prime}\right|^{\alpha}$ is the Jacobian for $f$ and $\mu$. The number $\alpha$ is called the exponent of the conformal measure.

Conformal measures were introduced in holomorphic iterations by D. Sullivan [S], who proved the existence of at least one such measure on $J$ by Patterson's method borrowed from Kleinian group theory. M. Denker and M. Urbański introduced in [DU1] the essential Hausdorff dimension (see the definition above) and proved (up to one gap filled in in [P1]) that there exists a smallest exponent $\alpha_{0}$ for which a conformal measure exists and

$$
\alpha_{0}=\operatorname{HD}_{\text {ess }}(f)
$$

A question raised in [DU1, Shi] and later in [P2] is whether for every $f$

$$
\operatorname{HD}_{\text {ess }}(J)=\operatorname{HD}(J) \text {. }
$$

The answer is for example positive if no critical point in $J$ is reccurrent, proved by M. Urbański in [U].

One considers also the so-called Minkowski dimension: lower and upper (other names: box dimension, limit capacity) defined as follows:

For $X$ a closed subset of a compact metric space the lower (upper) Minkowski dimension is

$$
\underline{\operatorname{Cap}}(X)(\overline{\operatorname{Cap}}(X)):=\liminf _{\varepsilon \rightarrow 0}(\limsup ) \frac{\log \mathcal{N}(\varepsilon)}{-\log \varepsilon}
$$

where $\mathcal{N}(\varepsilon)$ is the minimal number of discs of radius $\varepsilon$ whose union covers $X$.

It is clear and well known that $\operatorname{HD}(X) \leq \operatorname{Cap}(X) \leq \overline{\operatorname{Cap}}(X)$.

We call a periodic point $z=f^{n}(z) \in J(f)$ parabolic if $\left(f^{n}\right)^{\prime}(z)$ is a root of unity.

Notation. Write $\operatorname{Crit}(f)=\left\{c \in \overline{\mathbb{C}}: f^{\prime}(c)=0\right\}$ for the set of all critical points. Denote the set of all $f$-critical points in $J$ whose forward trajectories do not meet other critical points, by Crit'.

We prove the following:

Theorem A. Let $f: \overline{\mathbb{C}} \rightarrow \overline{\mathbb{C}}$ be a rational map and $\mu$ be an $\alpha$-conformal measure on the Julia set $J$. Assume that for every $f$-critical point $c \in \mathrm{Crit}^{\prime}$, for every ${ }^{1}$ $Q>0$ there exists $C_{Q}>0$ such that for every $n \geq 0$

$$
\left|\left(f^{n}\right)^{\prime}(f(c))\right| \geq C_{Q} \exp Q \sqrt{n} .
$$

Then

\footnotetext{
${ }^{1}$ Actually a specific constant $Q$ depending on $f$ is sufficient.
} 
1. $\alpha \geq \operatorname{HD}(J(f))$; hence $\operatorname{HD}_{\text {ess }}(J)=\alpha_{0}=\operatorname{HD}(J)$.

2. If one assumes also that there are no parabolic periodic points in $J(f)$ then $\alpha \geq \overline{\operatorname{Cap}}(J(f))$; hence $\operatorname{HD}_{\text {ess }}(J)=\alpha_{0}=\operatorname{HD}(J)=\underline{\operatorname{Cap}}(J)=\overline{\operatorname{Cap}}(J)$.

To prove Theorem A we control the $\mu$-measure versus diameter in power $\alpha$ for components of consecutive pre-images of an arbitrary, large, disc $B$ : Comp $f^{-1}(B)$, $\operatorname{Comp} f^{-1}\left(\operatorname{Comp} f^{-1}(B)\right), \ldots$. The aim is to prove $\mu(B(x, r)) \geq r^{\alpha^{\prime}}$ for an arbitrary $\alpha^{\prime}>\alpha$, every $x \in J$ and sufficiently many $r$ 's. An important point is the inequality

$$
\frac{\mu\left(\operatorname{Comp} f^{-1}(A)\right)}{\mu(A)} \geq \operatorname{Const}\left(\frac{\operatorname{diam} \operatorname{Comp} f^{-1}(A)}{\operatorname{diam} A}\right)^{\alpha},
$$

for $A$ in a neighbourhood of a critical value, $\operatorname{Comp} f^{-1}(A)$ a component of $f^{-1}(A)$ near a critical point.

Assuming the non-recurrence of critical points Urbański $[\mathrm{U}]$ made an estimate in both directions. We need only its easy "half" above so we do not need the non-recurrence.

Recently E. Prado proved ergodicity of every conformal measure for every nonrenormalizable $z \mapsto z^{2}+c$ with $c$ not in the main cardioid. The ideas go back to [Guckenheimer] and [BL]. The same procedure proves ergodicity in our case. Namely in Section 3 we prove

Theorem B. Let $f: \overline{\mathbb{C}} \rightarrow \overline{\mathbb{C}}$ be a rational map, not expanding on its Julia set $J$, and $\mu$ be an $\alpha$-conformal measure on $J$. Assume that for every $c \in \mathrm{Crit}^{\prime}$

$$
\sum_{n=0}^{\infty}\left|\left(f^{n}\right)^{\prime}(f(c))\right|^{-1}<\infty
$$

Then the number of ergodic components of $\mu$ does not exceed the number of $f$-critical points in $J$ and of parabolic periodic orbits.

Corollary. If there is one $f$-critical point in $J$ for $f$ as in Theorem $\mathrm{B}$ and no parabolic periodic points then every conformal measure on $J$ is ergodic.

Notation. Let $\nu:=\sup \left\{\right.$ multiplicity of $f^{n}$ at $\left.c: n \geq 1, c \in \operatorname{Crit}(f) \cap J\right\}$. Considering $f^{n}$ above rather than $f$ makes a difference if the forward orbit of one critical point hits another critical point. As it cannot happen $f^{n}(c)=c$ for $c \in \operatorname{Crit}(f) \cap J$, otherwise $c$ would be a sink, we get $\nu \leq \operatorname{deg}(f) \sharp \operatorname{Crit}(f)=\operatorname{deg}(f)(2 \operatorname{deg}(f)-2)$.

We shall write $\hat{t}:=\sup \left\{t \geq 0: f^{t}(\operatorname{Crit}(f) \cap J) \cap \operatorname{Crit}(f) \neq \emptyset\right\}$.

In Section 4 we shall prove the following

Theorem C. Let $f: \overline{\mathbb{C}} \rightarrow \overline{\mathbb{C}}$ be a rational map and $\mu$ be an $\alpha$-conformal measure on the Julia set $J=J(f)$ not having atoms at critical points ${ }^{2}$. Assume that there are no parabolic periodic points in $J(f)$ and that there exists $C>0$ such that for every $n \geq 1$ for every $c \in$ Crit' $^{\prime}$

$$
\int \frac{d \mu}{\operatorname{dist}\left(x, f^{n}(c)\right)^{(1-1 / \nu) \alpha}}<C^{-1}
$$

and there exists $\Lambda>1$ such that

$$
\left|\left(f^{n}\right)^{\prime}(f(c))\right| \geq C \Lambda^{n} \quad \text { for every } n \geq 0 .
$$

\footnotetext{
${ }^{2}$ Added in revision: In the preprint version this substantial assumption was missing. I owe the correction to J. Graczyk.
} 
Then there exists an $f$-invariant probability measure on $J$ absolutely continuous with respect to $\mu$, (pacim). ${ }^{3}$

We prove this theorem by proving that there is a uniform bound for iteration of the Perron-Frobenius-Ruelle operator $\mathcal{L}$ with potential $-\alpha \log \left|f^{\prime}\right|$ on the constant function equal to 1

$$
\mathcal{L}^{n}(1)(x) \leq C_{1}+C_{2} \sum_{c \in \operatorname{Crit}(f) \cap J} \sum_{j=0}^{\infty} \gamma^{j} \frac{\left|\left(f^{j-\hat{t}}\right)^{\prime}\left(f^{\hat{t}+1}(c)\right)\right|^{-\alpha / \nu}}{\operatorname{dist}\left(x, f^{j}(f(c))\right)^{(1-1 / \nu) \alpha}}
$$

For $j-\hat{t} \leq 0$ we write in this formula 1 instead of the above derivative. $\gamma$ is arbitrary greater than 1 .

Theorem $\mathrm{C}$ is analogous to the Collet-Eckmann theorem [CE] on the existence of pacim for maps of interval. There exists a theorem stronger than Collet-Eckmann's by Nowicki and van Strien [NS, N]. Nowicki and van Strien proved the existence of pacim assuming instead of (0.6) only (for $\alpha=1, f$ a non-flat $S$-unimodal map of the interval)

$$
\sum_{n=1}^{\infty}\left|\left(f^{n}\right)^{\prime}(f(c))\right|^{-1 / \nu}<\infty
$$

Unfortunately we do not know how to get rid of the factor $\gamma^{j}$ in (0.7), introduced to swallow constants from the distortion estimates.

We do not know how to get rid of the assumption (0.5). Maybe it always holds? At least it holds in such a highly non-trivial case as $J(f)=\overline{\mathbb{C}}$ with the standard spherical area conformal measure with the exponent 2 . There are several papers on the existence of pacim in the complex case $J(f)=\overline{\mathbb{C}}$ if critical points are nonrecurrent. The only one, to my knowledge, in which recurrence of critical points is allowed, is $[R]$. It would be interesting to understand connections of $[R]$ with our paper.

The strategy of the Proof of Theorem $\mathrm{C}$ is the same as in [CE, NS] and [N]. A new element is that we learned how to cope with distortion in the complex case. Similarly as in the interval case we rely on Koebe distortion estimate for iterates $f^{-n}$ and the non-flatness of $f$ at critical points. This allows us to prove also Theorem A (see $\S 2$, Def.2.3).

In Appendix we prove the following

Theorem D. For every non-renormalizable quadratic polynomial $z \mapsto z^{2}+c$, with $c$ not in the main cardioid in the Mandelbrot set, the assertion 1. of Theorem A holds.

The geometry of Proof of Theorem D is surprisingly similar to that of Theorem $\mathrm{A}$, this is the reason we included it in this paper. We do not assume anything on the growth of $\left|\left(f^{n}\right)^{\prime}(c)\right|$. Instead we use the Markov structure of the puzzle.

Theorem D is rather an easy corollary from a deep theory by M. Jakobson, G. Świątek, J. Graczyk [GS1] and M. Lyubich [L]. The holomorphic side of it grew up from J.-Ch. Yoccoz ideas $[\mathrm{Y}]$.

\footnotetext{
${ }^{3}$ Added in revision: An estimate by $C^{-1}\left(\Lambda^{\prime}\right)^{\alpha n / \nu}$ in (0.5) for an arbitrary $1<\Lambda^{\prime}<\Lambda$ would be sufficient for Theorem $\mathrm{C}$ to hold. We need the majorating function in (0.7) to be $\mu$-integrable. To have this weaker condition will be crucial in the subsequent paper [P3].
} 
Note. In a preliminary version of the paper this theorem was proved under the assumption that so-called cascades of central returns are short enough that all annuli between consecutive puzzle pieces in the principal nest (see Appendix for the terminology) have moduli bounded away from 0 . That it is possible to cope with the general non-renormalizable case by taking a non-principal nest of critical puzzle pieces was suggested to me by J. Graczyk and M. Lyubich.

Notation. Const will denote various positive constants which may change from one formula to another, even in one string of estimates.

Acknowledgements. I want to thank J. Graczyk, M. Lyubich (see Note above) and J.-Ch. Yoccoz for discussions which helped me to figure out Theorem D, M. Urbański for a fruitful criticism of a very first draft of Theorem A, finally J. Graczyk and M. Urbański for corrections in Proof of Theorem B.

\section{Controlling Pre-Images. Distortion}

Lemma 1.1. For every integer $K \geq 0$ and $0<\lambda<1$ the following hold:

1. For every $\varepsilon>0$ there exists $\delta_{0}>0$ such that for every disc $B=B(x, \delta)$ with $\delta \leq \delta_{0}, x \in J$, for every $n \geq 0$ and every connected component $W=$ Comp $f^{-n}(B(x, \delta))$ such that $\left.f^{n}\right|_{W}$ has at most $K$ critical points counted with multiplicities, for every component $W^{\prime}=\operatorname{Comp} f^{-n}\left(B^{\prime}\right)$ in $W$, for the disc $B^{\prime}=$ $B(x, \lambda \delta))$, we have

$$
\operatorname{diam} W^{\prime} \leq \varepsilon
$$

2. $\operatorname{diam} W^{\prime} \rightarrow 0$ for $n \rightarrow \infty$ uniformly (i.e. independently of the choices of $B$ and $\left.W^{\prime}\right)$.

Proof. This is a standard lemma, for one of its variants we could refer to [M] (though here we assume $x \in J$ rather than that $x$ is far from parabolic points or sinks, otherwise 2. would be false for $B(x, \delta)$ in a Siegel disc or a Herman ring). Nevertheless for the completeness we shall prove it:

Let $B_{n}=B\left(x_{n}, \delta_{n}\right)$ be a sequence of discs with $x_{n} \in J$. Consider also a sequence of components $W_{n}=\operatorname{Comp} f^{-k_{n}}\left(B_{n}\right)$ with $k_{n} \rightarrow \infty$, as $n \rightarrow \infty$ and number of critical points of each $f^{k_{n}}$ on $W_{n}$ bounded by $K$. Then for each $n$ there exists $L=L(n): 0 \leq L \leq K$ such that there is no critical value of $\left.f^{k_{n}}\right|_{W_{n}}$ in

$$
P(n)=: B\left(x_{n}, \delta_{n}\left(\lambda+(1-\lambda) \frac{L+1}{K+1}\right)\right) \backslash B\left(x_{n}, \delta_{n}\left(\lambda+(1-\lambda) \frac{L}{K+1}\right)\right) .
$$

Let $W_{n}^{\prime}$ be associate to $W_{n}$ as in the statement of the lemma. Suppose that $\operatorname{diam} W_{n}^{\prime} \nrightarrow 0$; i.e. there exists $\eta>0$ such that $\operatorname{diam} W_{n}^{\prime}>\eta$.

To prove 1 . suppose that $\delta_{n} \rightarrow 0$.

Denote

$$
\begin{gathered}
W_{n}^{(1)}=: \operatorname{Comp} f^{-k_{n}}\left(B\left(x_{n}, \delta_{n}\left(\lambda+(1-\lambda) \frac{L(n)}{K+1}\right)\right)\right), \\
W_{n}^{(2)}=\operatorname{Comp} f^{-k_{n}}\left(B\left(x_{n}, \delta_{n}\left(\lambda+(1-\lambda) \frac{L(n)+1}{K+1}\right)\right)\right)
\end{gathered}
$$

the components containing $W_{n}^{\prime}$,

$$
P_{n}=: W_{n}^{(2)} \backslash W_{n}^{(1)}
$$


and for every $0 \leq m \leq k_{n}, i=1,2$,

$$
W_{n, m}^{(i)}=: f^{k_{n}-m}\left(W_{n}^{(i)}\right), \quad P_{n, m}=: f^{k_{n}-m}\left(P_{n}\right)=W_{n, m}^{(2)} \backslash W_{n, m}^{(1)} .
$$

Let, for each $n$, the number $m=m(n) \leq k_{n}$ be the least integer such that

$$
\operatorname{diam} W_{n, m}^{(1)} \geq \min \left(\eta, \inf _{c_{1}, c_{2} \in \operatorname{Crit}(f), c_{1} \neq c_{2}} \operatorname{dist}\left(c_{1}, c_{2}\right)\right) .
$$

So for every $0 \leq t<m(n)$ the set $P_{n, t}$ is a topological annulus. That is because at each step back by $f^{-1}$ from $P_{n, t-1}$ to $P_{n, t}$ there is at most one branch point for $f^{-1}$ from $W_{n, t-1}^{(i)}$ to $W_{n, t}^{(i)}, i=1,2$.

All $P_{n, m(n)-1}$ 's have moduli bounded from below by $2^{-K}(1-\lambda) \frac{1}{K+1}$. So by Montel's Theorem there is a topological (maybe not geometric) annulus $P$ contained in all $P_{n_{s}, m\left(n_{s}\right)-1}$ 's for a subsequence $n_{s}$, which bounds a disc $D$ containing a point $y \in J$. So $D \subset W_{n_{s}, m\left(n_{s}\right)-1}^{(2)}$; hence $f^{m\left(n_{s}\right)-1}(D) \subset B\left(x, \delta_{n}\right)$. By $\delta_{n} \rightarrow 0$ we have also $m(n) \rightarrow \infty$. Thus we have arrived at a contradiction with $y \in J$. This proves 1.

To prove 2. observe that if for every $n, \delta_{n} \leq \delta$ and $\delta$ is small enough then

$$
\operatorname{diamComp} f^{-n}(B(x, \lambda \delta))
$$

is small for all $n$. This allows us to repeat the above proof that

$$
\operatorname{diam} \operatorname{Comp} f^{-n}\left(B\left(x_{n}, \lambda^{2} \delta\right)\right) \nrightarrow 0
$$

leads to a contradiction.

(We do not need now $m(n)$ 's so we do not need $\delta_{n} \rightarrow 0$.)

Now we shall discuss how annuli control distortion.

We start with a variant of Koebe's Distortion Lemma:

Lemma 1.2. Given $\varepsilon<\operatorname{diam} \overline{\mathbb{C}}$ there exists a constant $C=C(\varepsilon)$ such that for every $0<\lambda<1$, every disc $B(z, \delta) \subset \overline{\mathbb{C}}$ with $\delta \leq \varepsilon$, every holomorphic univalent mapping $g: B(z, \delta) \rightarrow \overline{\mathbb{C}}$ for which

(i) $\operatorname{diam} g(B(z, \delta)) \leq \varepsilon \quad$ or at least (ii) $\operatorname{diam}(\overline{\mathbb{C}} \backslash g(B(z, \delta))) \geq \varepsilon$, and every $x, y \in B(z, \lambda \delta)$ it holds:

$$
\begin{gathered}
\left|g^{\prime}(x)\right| /\left|g^{\prime}(z)\right|<C(1-\lambda)^{-1}, \quad\left|g^{\prime}(z)\right| /\left|g^{\prime}(x)\right|<C(1-\lambda)^{-1}, \\
\left|g^{\prime}(x)\right| /\left|g^{\prime}(y)\right|<C(1-\lambda)^{-2} .
\end{gathered}
$$

Proof. Recall that we consider $\left|g^{\prime}\right|$ and diameters in the spherical metric. In the standard Koebe Lemma in the complex plane the exponents are -3 rather than -1 in the first inequality and -4 rather than -2 in the last one $[\mathrm{H}]$. Here assuming (i) we can change coordinates and have $g$ from the unit disc in $\mathbb{C}$ into a given bounded region. Then one obtains the exponent -1 in the first inequality (use Cauchy's formula for $g^{\prime}$, pass to absolute values, you arrive at Poisson kernel). If only (ii) is assumed we choose two points $a, b \in \overline{\mathbb{C}} \backslash g(B(z, \delta))$ far from $g(x), g(y)$ and change coordinates moving $a, b$ to $0, \infty$, next apply a branch of the square root and again a homography ending with a new $g(B(z, \delta))$ bounded in $\mathbb{C}$, the bound depending on $\varepsilon$.

Later for $g$ holomorphic on a neighbourhood $U$ of $X$ the number $\sup _{x, y \in X} \frac{\left|g^{\prime}(x)\right|}{\left|g^{\prime}(y)\right|}$ will be called the distortion of $g$. We shall denote it by $\operatorname{Distor}(g, X)$. For $\operatorname{diam} U \leq \varepsilon$ being an arbitrary constant less than diam $\overline{\mathbb{C}}$, the number $\sup _{g} \operatorname{Distor}(g, X)$ over all 
univalent holomorphic $g: U \rightarrow \overline{\mathbb{C}}$ such that diam $g(U) \leq \varepsilon$ will be denoted by Distor $_{U, X}$.

We shall need also the following:

Lemma 1.3. For every rational function $f: \overline{\mathbb{C}} \rightarrow \overline{\mathbb{C}}$ there exists $C_{3}>0$ such that for every connected $A \subset \overline{\mathbb{C}}$ and $\operatorname{Comp} f^{-1}(A)$

$$
\frac{\operatorname{diam} \operatorname{Comp} f^{-1}(A)}{\operatorname{diam} A} \leq C_{3} \inf \left\{\left|f^{\prime}(x)\right|^{-1}: x \in \operatorname{Comp} f^{-1}(A)\right\}
$$

and for $A_{1} \subset A_{2} \subset \overline{\mathbb{C}}$ connected and components $\operatorname{Comp} f^{-1}\left(A_{1}\right) \subset \operatorname{Comp} f^{-1}\left(A_{2}\right)$

$$
\frac{\operatorname{diam}\left(\operatorname{Comp} f^{-1}\left(A_{1}\right)\right)}{\operatorname{diam}\left(\operatorname{Comp} f^{-1}\left(A_{2}\right)\right)} \geq C_{3}^{-1} \frac{\operatorname{diam}\left(A_{1}\right)}{\operatorname{diam}\left(A_{2}\right)} .
$$

Proof. For large $A$ (1.2) is trivial. Small $\operatorname{Comp} f^{-1}(A)$ far from Crit is taken care of in Lemma 1.2. If $\operatorname{Comp} f^{-1}(A)$ is small close to $c \in \operatorname{Crit}(f)$ (1.2) is easily calculable after a holomorphic change of coordinates in a neighbourhood of $c$ so that $f$ becomes $z \mapsto z^{\nu(c)}$. The inequality (1.3) follows from (1.2). Namely for $A=A_{2}$ we can replace the right side of (1.2) by $\frac{\operatorname{diamComp} f^{-1}\left(A_{1}\right)}{\operatorname{diam} A_{1}}$.

Now we give a $K$-critical variant of Lemma 1.2 :

Lemma 1.4. In the situation of Lemma 1.1 there exist also constants $L>0$ depending only on $K$ and $C_{4}>0$ depending on $K, \varepsilon$ such that for every $x \in W^{\prime}$ we have

$$
\operatorname{diam} W^{\prime} \leq C_{4}(1-\lambda)^{-L}\left|\left(f^{n}\right)^{\prime}(x)\right|^{-1} \operatorname{diam} B^{\prime} ;
$$

for every $B^{1} \subset B^{2} \subset B^{\prime}$ and components $W^{1} \subset W^{2} \subset W^{\prime}$ of their $f^{-n}$-preimages we have

on the other hand

$$
\frac{\operatorname{diam} W^{1}}{\operatorname{diam} W^{2}} \geq C_{4}^{-1}(1-\lambda)^{L} \frac{\operatorname{diam} B^{1}}{\operatorname{diam} B^{2}}
$$

$$
\frac{\operatorname{diam} W^{1}}{\operatorname{diam} W^{2}} \leq C_{4}(1-\lambda)^{-L}\left(\frac{\operatorname{diam} B^{1}}{\operatorname{diam} B^{2}}\right)^{2^{-K}} .
$$

Proof. Similarly as in Proof of Lemma 1.1 we divide $B \backslash B^{\prime}$ into $K+2$ annuli and choose that one which does not contain critical values for $\left.f^{n}\right|_{W}$ but not the most external one. (This is why we took $K+2$ rather than $K+1$.) So from the beginning we assume that there are no critical values for $\left.f^{n}\right|_{W}$ in $P=B \backslash B^{\prime}$ whose modulus satisfies $\bmod P \geq \frac{1-\lambda}{K+2}$. As we removed the most external annulus we know by Lemma 1 part 1 , that for $B$ small, all $f^{j}(W), j=0,1, \ldots, n$, are small so Lemma 1.2 will be applicable.

Let $0<m_{1}<m_{2}<\ldots<m_{K^{\prime}} \leq n\left(K^{\prime} \leq K\right)$ be all consecutive integers $m$ such that $f^{n-m}\left(W^{\prime}\right)$ contain $f$-critical points. If $m_{K^{\prime}}<n$ consider also $m_{K^{\prime}+1}=n+1$. Then for each $m_{t}, t \leq K^{\prime}$,

$$
\operatorname{diam} f^{n-m_{t}}\left(W^{\prime}\right) \leq C_{3} \operatorname{diam} f^{n-m_{t}+1}\left(W^{\prime}\right) \mid f^{\prime}\left(\left.f^{n-m_{t}}(x)\right|^{-1}\right.
$$

by Lemma 1.3 (the inequality (1.2)).

We need to estimate distortions of $f^{m_{t}-m_{t-1}-1}$ on $f^{n-m_{t}+1}\left(W^{\prime}\right)$ for each $t$ such that $m_{t-1}-m_{t}>1$. It is clear that each annulus $f^{m}\left(W \backslash W^{\prime}\right)$ has modulus at least $a=(1-\lambda) 2^{-K} /(K+2)$ (see an explanation later) but this is not enough because this annulus need not be a geometric annulus. For $a$ small one has only the 
distortion estimate in the encircled domain by Const $\exp (2 / a)$. Take for example $D$ a unit disc and $D^{\prime}:=[-\tau, \tau]$ for $\tau<1, \tau \approx 1$. Then $\bmod \left(D \backslash D^{\prime}\right)$ is of order Const $\log (1 /(1-\tau))$ but the distortion on $D^{\prime}$ of say the Koebe function $\frac{z}{(1-z)^{2}}$ is of order $1 /(1-\tau)^{2}$ (in the spherical metric).

Fortunately for modulus not too small this surprise does not happen. It is easy to see that for every $a>0$ there exists $\vartheta(a)$ such that if $\bmod \left(D \backslash D^{\prime}\right)>a$ then Distor $_{D, D^{\prime}}<\vartheta(a)$.

We obtain now the estimate of Distor $f^{n-m}(W), f^{n-m}\left(W^{\prime}\right)$ for $m=m_{1}-1, m_{2}-1, \ldots$ by induction making the following trick:

Cover (this is easy) $B^{\prime}$ by a family of $\operatorname{discs} \mathcal{B}=\left\{B\left(z_{j}, u_{j}\right)\right\}$ such that for every $j$ we have $B\left(z_{j}, 2 u_{j}\right) \subset B$ and

$$
\sharp \mathcal{B} \leq \text { Const } \log (1 /(1-\lambda)) .
$$

Consider for each $m \leq n$ the family $\mathcal{B}_{m}$ of components of $f^{-m}(b)$, for all $b \in \mathcal{B}$, intersecting $f^{n-m}\left(W^{\prime}\right)$, and denote by $2 \mathcal{B}_{m}$ the family of corresponding components of $f^{-m}(b)$ for $b=B\left(z_{j}, 2 u_{j}\right)$. For each $V \in \mathcal{B}_{m}$ and corresponding $V^{\prime} \in 2 \mathcal{B}_{m}$, i.e. obtained for the same $j$ and containing $V$, either $\bmod \left(V^{\prime} \backslash V\right)=\bmod \left(f\left(V^{\prime}\right) \backslash f(V)\right)$, which is the case if $m \neq m_{1}, m_{2}, \ldots, m_{K^{\prime}}$, or $\bmod \left(V^{\prime} \backslash V\right) \geq \frac{1}{\nu} \bmod \left(f\left(V^{\prime}\right) \backslash f(V)\right)$ where $\nu$ majorates the multiplicities of $f$ at critical points.

Observe also that

$$
\sharp \mathcal{B}_{m} \leq \gamma \sharp \mathcal{B}_{m-1}
$$

where $\gamma=\nu$ or 1 depending as $m$ is one of the numbers $m_{1}, m_{2}, \ldots, m_{K^{\prime}}$ or not.

We conclude that for each $m$, in particular for $m=m_{t}-1, t=1, \ldots, K^{\prime}+1$,

$$
\text { Distor }_{f^{n-m}(W), f^{n-m}\left(W^{\prime}\right)} \leq\left(\left(\vartheta\left(2^{-K}\right)\right)^{K}\right)^{\sharp \mathcal{B}_{m}} \leq \operatorname{Const}(1-\lambda)^{-L^{\prime}}
$$

for an appropriate $L^{\prime}$ by (1.7) and the composition of (1.8) for all $m$ 's. This applied to $g=f^{m_{t}-m_{t-1}-1}$ on $f^{n-m_{t}+1}\left(W^{\prime}\right)$ for each $t$, together with (1.6), gives (1.4) with $L=(K+1) L^{\prime}$.

The estimate (1.5) follows similarly: For $m=m_{t}, t=1, \ldots, K^{\prime}$, take $A_{i}$ in (1.3) being $f^{m_{t}+1}\left(W^{i}\right)$ and $\operatorname{Comp} f^{-1}\left(A_{i}\right)=f^{m_{t}}\left(W^{i}\right)$ and use (1.3). For $m=m_{t}-1, t=$ $1, \ldots, K^{\prime}+1$, use $(1.9)$.

In $\left(1.5^{\prime}\right)$ the difference in the proof is for $m=m_{t}, t=1, \ldots, K^{\prime}$. The root of the diameter is taken then, according to the multiplicity of the critical point.

One can change the assumptions of Lemma 1.4 to obtain

Lemma 1.5. ${ }^{4}$ For every $\varepsilon>0$ and integer $K \geq 0$ there exist positive constants $L, C_{4}$ such that for every $\delta>0,0<\lambda<1$ and for every $B, W, B^{\prime}, W^{\prime}, B^{i}, W^{i}, i=$ 1,2 , as in Lemma 1.1 for which

1. $\operatorname{diam}\left(\overline{\mathbb{C}} \backslash f^{j}(W)\right) \geq \varepsilon, j=0,1, \ldots, n$,

2. each $f^{j}(W)$ contains at most one critical point (maybe multiple),

the estimates (1.4),(1.5) and $\left(1.5^{\prime}\right)$ hold.

Proof. Proof is the same as for Lemma 1.4 except at the beginning. We have no means to prove 1 . and 2. above (which we need in order to know that all $f^{j}(W)$ are simply-connected). This is the reason we just assumed 1 . and 2.

\footnotetext{
${ }^{4}$ Added in revision: This modification of Lemma 1.4 was missing in the preprint version. But in $\S 2$, in applying Def. 2.3, we shall keep $\delta$ fixed and maneuver with $\lambda \approx 1$, so this modification will be of use. See also the recent paper: F. Przytycki, S. Rohde: Porosity of Collet-Eckmann Julia sets, preprint, 1996.
} 


\section{Conformal measures. Proof of Theorem A}

We shall need the following technical facts:

Lemma 2.1 (see [P1] or [DPU, Rule I]). For every $f: \mathbb{C} \rightarrow \mathbb{C}$ a rational mapping on the Riemann sphere there exists $\kappa>0$ such that if for a critical point $c \in J(f)$ and $n>0$ we have $\operatorname{dist}\left(f^{n}(c), c\right)<\varepsilon$ then $n \geq \kappa \log 1 / \varepsilon$.

Lemma 2.2 (see [DPU]). There exist $\theta>1, \rho>0$ such that for every $x \in J(f), n$ $>0$ for every disc $B=B(x, \delta)$ and every component $W$ of $f^{-n}(B)$ one has $\operatorname{diam} W \leq \theta^{n} \delta^{\rho}$.

(Lemma 2.2 is an elementary but a very non-trivial fact. It follows from Rule II, see [DPU]. One can take $\rho=2^{-2 \operatorname{deg} f+2}$.)

Let us pass now to the definition which provides a basic idea of the paper:

Definition 2.3. Fix a series of positive numbers $\sum_{n=1}^{\infty} b_{n}=1 / 2$. For every disc $B=B(x, r)$ denote by $B_{[n}$ the $\operatorname{disc} B\left(x,\left(\prod_{j=1}^{n}\left(1-b_{j}\right)\right) r\right)$. We call $n$ the essential critical time for $B$ and for a sequence of compatible components $W_{t}=$ Comp $f^{-t}\left(B_{[t}\right)$, compatible means $f\left(W_{t}\right) \subset W_{t-1}$, if there exists a critical point $c \in W_{n}$. The time $n$ is called exposed if $c \in$ Crit' $^{\prime}$.

The issue is that in backward iteration, for consecutive compatible components Comp $f^{-n}\left(B_{[n}\right)$, if there is no critical $c$ as above, we remove $B_{[n} \backslash B_{[n+1}$. In the sequence of all essential critical times, for the first one, $n$, we have the "isolating" annulus $B_{[n-1} \backslash B_{[n}$, of modulus $\geq b_{n}$, which allows us to estimate distortion for the appropriate branch of $f^{-(n-1)}$ on $B_{[n}$ and use (0.4). See Figure 1. In fact for technical reasons we shall always consider a $(K+1)$-th essential critical time. We should take into account also the possibility that the forward orbit of one critical point hits another critical point.

Remark 2.4. Note that, for $n$ being the $(K+1)$-th critical time, $W_{n}$ contains only one critical point. Moreover this holds for every smaller essential critical time. In consequence we are in the position to use the assertion of Lemma 1.4, in particular the estimate (1.4) for $B=B_{[n-1}, B^{\prime}=B_{[n}, W=f\left(W_{n}\right), W^{\prime}=W_{n-1}$; formally we can use Lemma 1.5 (if also its condition 2. holds).

We prove this claim by induction over essential critical times. Suppose $B_{[m}$ captures a critical point $c \in \mathrm{Crit}^{\prime}$. Then each $f^{i}\left(W_{m-1}\right), 0<i \leq m-1$, contains at most one critical point by the induction hypothesis. We apply Lemma 1.5; hence (1.4) gives $\operatorname{diam} f\left(W_{m}\right) \leq$ Const $b_{m}^{-L}\left|\left(f^{m-1}\right)^{\prime}(f(c))\right|^{-1}$. which is small by $(0.4)$. (We can assume $m$ is large; for small $m$ it is clear directly that diam $W_{m}$ is small if $r$ is small enough.) So $W_{m}$ has small diameter. Hence it contains only one critical point. Also all $W_{m+j}, j=1,2, \ldots, \hat{t}$, have small diameter, so each one contains at most one critical point ( $\hat{t}$ defined in Introduction). We have performed the induction step because the next critical time will be again exposed (i.e. $c \in \mathrm{Crit}^{\prime}$ ). (In Sec. 3. the reader will find related estimates; see also Step 3 of Proof of Theorem A.)

Condition 2. of Lemma 1.5 holds for $r$ small enough that for each $x \in J$ the set $\mathbb{C} \backslash B(x, r)$ contains at least two different points of an a priori fixed periodic orbit. Then each $f^{-n}(B(x, r))$ also misses two points of this orbit; hence 2 . holds with $\varepsilon$ being the minimal distance between any pair of distinct points of this orbit.

Proof of Theorem A. We shall work under the assumption that there are no parabolic periodic points. Only at the last Step 6 shall we comment on the situation where there are some. 


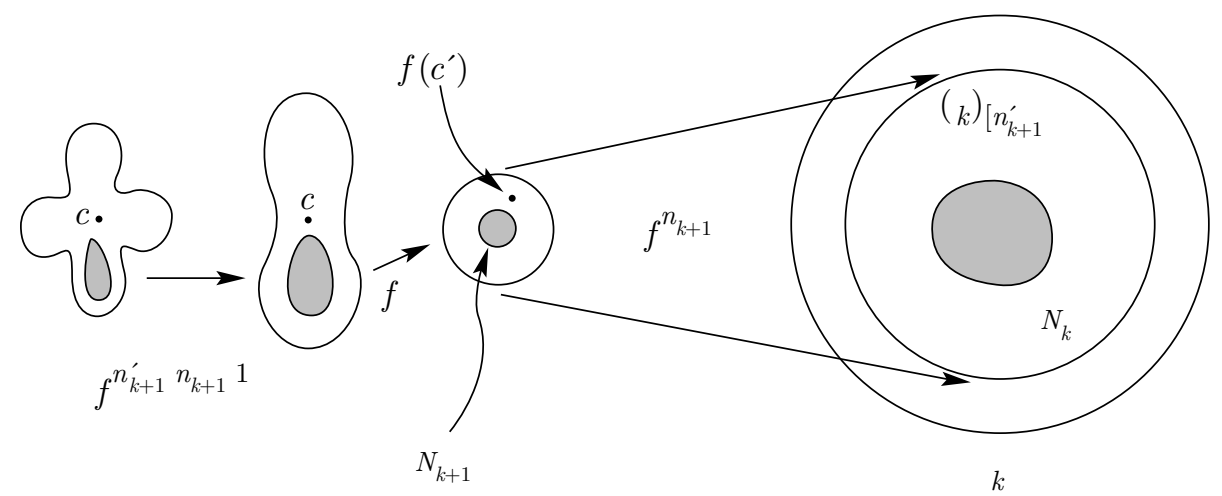

Figure 1

The crucial estimate will be

$$
\mu(B(x, r)) \geq r^{\alpha^{\prime}} .
$$

In order to cope with Minkowski dimension (Cap) it is sufficient to prove the following:

For every $\alpha^{\prime}>\alpha, \beta>1$ there exists $\varepsilon_{0}>0$ such that for every $x \in J$ and $0<\varepsilon<\varepsilon_{0}$ there exists $r: \varepsilon^{\beta}<r<\varepsilon$ for which (2.1) is satisfied.

Indeed, having proved $(*)$ we obtain for every $\varepsilon<\varepsilon_{0}$, with the help of the Besicovitsch covering theorem [Gu], a covering $\mathcal{P}$ of $J$ with discs $B\left(z_{j}, r_{j}\right)$ of radii $r_{j}$ between $\varepsilon^{\beta}$ and $\varepsilon$, satisfying (2.1), such that each point belongs to at least $M$ such discs for a constant $M$ depending only on the metric on $\overline{\mathbb{C}}$. Hence

$$
\sum_{j} r_{j}^{\alpha^{\prime}} \leq \sum_{j} \mu\left(B\left(x_{j}, r_{j}\right)\right) \leq M \text {. }
$$

So $M \geq \sum_{j} \varepsilon^{\beta \alpha^{\prime}}=\sharp \mathcal{P} \varepsilon^{\beta \alpha^{\prime}}$; hence $\frac{\log \sharp \mathcal{P}}{-\beta \alpha^{\prime} \log \varepsilon} \leq \gamma$ where $\gamma>1$ is arbitrarily close to 1 for $\varepsilon$ small enough. Hence $\overline{\text { Cap }} \leq \beta \alpha^{\prime}$. Due to the freedom of choice of $\beta, \alpha^{\prime}$ we obtain $\overline{\mathrm{Cap}} \leq \alpha$.

(This is a standard consideration related to Frostman's lemma, see [PUbook].)

Step 1. Basic construction. Fix arbitrary $\alpha^{\prime}>\alpha$. Fix $\xi>10$ and $\delta>0$, depending on $\xi$ and $\alpha^{\prime}$ and on constants depending on $f$ (the dependence will be explained later). Fix also $K=\sharp$ Crit $\cap J \leq 2 \operatorname{deg} f-2$.

The idea is to prove $\mu(U) \geq(\operatorname{diam} U)^{\alpha^{\prime}}$ for every $x, n$ and all the sets $U:=U_{n}$ which are components of $f^{-n}(B(x, \delta))$ such that $f\left(U_{n}\right)=U_{n-1}$. We do this more or less recurrently, for $n=1,2, \ldots$.

So fix arbitrary $x$ and $U_{n}$ as above. (This $x$ is not the one in (2.1)!)

We choose a sequence of integers $n_{j}, j=0,1, \ldots$, (and other objects) by induction as follows: $n_{0}=0$. Suppose that all $n_{j}$ for $j=0,1, \ldots, k$ are already chosen. Denote $N_{k}=\sum_{j=1}^{k} n_{j}$. Define $V_{k}:=B\left(z, \xi^{k+1} \operatorname{diam} U_{N_{k}}\right)$ with an arbitrary origin $z \in U_{N_{k}}$. We define $n_{k+1}^{\prime}$ as the $K+1$-th essential critical time for $V_{k}$ and for the sequence of components $\operatorname{Comp} f^{-n}\left(\left(V_{k}\right)_{[n}\right)$ such that $\operatorname{Comp} f^{-n}\left(\left(V_{k}\right)_{[n}\right) \supset U_{N_{k}+n}$. For each $c \in$ Crit $\cap J$ write $t(c)$ for the largest non-negative integer such that 
$c^{\prime}=f^{t(c)}(c) \in$ Crit. Finally define $n_{k+1}=n_{k+1}^{\prime}-t(c)-1$ where $c$ is the critical point appearing in the definition of the essential critical time $n=n_{k+1}^{\prime}$. (There is only one $c$ for $n$, cf. Remark 2.4.) See Figure 1.

Of course $n_{k+1}$ may not exist for certain $k$; i.e. there exist at most $K$ essential critical times. Then the sequence $n_{j}, j>0$, is of finite length $k$. Formally we set then $n_{k+1}=N_{k+1}=\infty$. Also a priori it is not clear that $n_{j}$ 's are positive (so that the construction makes sense). It will be proved in Step 3.

Step 2. Conformal measure versus diameter. Observe that for every $k \geq 0$ and $0 \leq$ $m \leq n_{k+1}$ by (1.4) applied to $B=\left(V_{k}\right)_{[m}, B^{\prime}=B\left(z, \operatorname{diam} U_{N_{k}}\right)$ with $z \in U_{N_{k}}$ as in the definition of $V_{k}, W^{\prime} \supset U_{N_{k}+m}$, one obtains

$$
\operatorname{diam} U_{N_{k}+m} \leq C_{4} 2^{L}\left|\left(f^{m}\right)^{\prime}(x)\right|^{-1} \operatorname{diam} U_{N_{k}} .
$$

When writing here 2 in place of $1 /(1-\lambda)$ we use the fact that $\xi$ is large; i.e. $U_{N_{k}}$ sits well inside $V_{k}$ hence also inside $\left(V_{k}\right)_{[m}$. (We do not need here the delicate part of $(1.4)$ when $\lambda \approx 1$.)

By integrating $\left|\left(f^{m}\right)^{\prime}(x)\right|^{-1}$ and using the assumption that $\mu$ is $\alpha$-conformal one obtains

$$
\left(\frac{\operatorname{diam} U_{N_{k}+m}}{\operatorname{diam} U_{N_{k}}}\right)^{\alpha} \leq\left(C_{4} 2^{L}\right)^{\alpha} \frac{\mu\left(U_{N_{k}+m}\right)}{\mu\left(U_{N_{k}}\right)} .
$$

In particular this holds for $m=n_{k+1}$.

Combining these inequalities we obtain for $N_{k} \leq n \leq N_{k+1}$

$$
\left(\frac{\operatorname{diam} U_{n}}{\operatorname{diam} U_{0}}\right)^{\alpha} \leq\left(C_{4} 2^{L}\right)^{\alpha(k+1)} \frac{\mu\left(U_{n}\right)}{\mu\left(U_{0}\right)}
$$

Hence using the fact that $\mu$ is positive on open sets (because every rational function maps every open set in the Julia set onto the Julia set after a few iterations) and the assumption that $U_{0}$ has a definite diameter $2 \delta$ we obtain

$$
\mu\left(U_{n}\right) /\left(\operatorname{diam} U_{n}\right)^{\alpha} \geq \operatorname{Const}\left(C_{4} 2^{L}\right)^{-k} .
$$

Step 3. Diameters of $U_{N_{k}}$ 's. We prove by induction, using (0.4) and distortion estimates, that $n_{k}$ 's are large and

$$
\operatorname{diam} U_{N_{k}} \leq \exp \left(-k^{2}\right) .
$$

So assume (2.3) for an arbitrary $k$. Then by definition $\operatorname{diam} V_{k} \leq 2 \xi^{k+1} \exp \left(-k^{2}\right)$. By Lemma 2.2 the components $\operatorname{Comp} f^{-m}\left(V_{k}\right) \supset U_{N_{k}+m}$ satisfy

$$
\operatorname{diamComp} f^{-m}\left(V_{k}\right) \leq \theta^{m} \xi^{\rho(k+1)} \exp \left(-\rho k^{2}\right)
$$

So by Lemma 2.1, to meet twice the same critical point (first with $\operatorname{Comp} f^{-i}\left(V_{k}\right)$ and next with $\operatorname{Comp} f^{-j}\left(V_{k}\right)$ for some $\left.0<i<j \leq n_{k+1}^{\prime}\right)$, it is necessary that for $l=\max (i, j)$

$$
n_{k+1}^{\prime} \geq j-i \geq \kappa \log \left(\theta^{l} \xi^{\rho(k+1)} \exp \left(-\rho k^{2}\right)\right)^{-1}=-\kappa l \log \theta-\kappa(k+1) \log \xi+\rho k^{2} .
$$

Replacing $l$ by $n_{k+1}^{\prime}$ we obtain

$$
n_{k+1}^{\prime} \geq \frac{-\kappa(k+1) \log \xi+\rho k^{2}}{1+\kappa \log \theta} \geq \sigma k^{2}
$$

for a constant $\sigma>0$ and $k \geq k_{0}$, for a constant $k_{0}$ resulting from the above formula. For every $k<k_{0}$ the estimate (2.3) holds automatically if the diameter of $U_{0}$, i.e. the constant $\delta$, is small enough. This follows from Lemma 1.1, part 1 . 
(Unlike in previous applications where we only needed $\varepsilon$ sufficiently small to have a definite constant for the distortion, for example $C(\varepsilon)$ in Lemma 1.2, we need here to maneuver with $\varepsilon$ because we need to use Lemma 1.2 in $k_{0}-1$ steps.)

For $n_{k+1}$ we obtain the same estimate as (2.4):

$$
n_{k+1} \geq \sigma k^{2}
$$

(with slightly smaller $\sigma$ by using the fact that $n_{k+1}^{\prime}-n_{k+1} \leq \sup _{c \in \operatorname{Crit} \cap J} t(c)$ are uniformly bounded. By the way we have proved that $n_{k}$ are positive for $k \geq k_{0}$. Again, for $k<k_{0}$ it is so if $\operatorname{diam} U_{0}$ is small enough).

We know now that there exists a critical point $c^{\prime} \in \operatorname{Comp} f^{-n_{k+1}-1}\left(\left(V_{k}\right)_{\left[\left(n_{k+1}+1\right)\right.}\right)$ (the component containing $U_{N_{k+1}+1}$ ), which satisfies (0.4). So by (1.4)

$$
\begin{aligned}
\operatorname{diam} U_{n_{k+1}} & \leq \operatorname{diamComp} f^{-n_{k+1}}\left(\left(V_{k}\right)_{\left[\left(n_{k+1}+1\right)\right.}\right) \\
& \leq \operatorname{diam}\left(V_{k}\right)_{\left[\left(n_{k+1}+1\right)\right.} \times C_{4} b_{n_{k+1}+1}^{-L} \times\left|\left(f^{n_{k+1}}\right)^{\prime}(f(c))\right|^{-1} .
\end{aligned}
$$

We could fix $b_{n}$ polynomially decreasing to 0 and then the latter expression can be further majorized by

$$
\text { Const } \exp \left(-k^{2}\right) \xi^{k+1} \exp \left(-\frac{1}{2} Q \sqrt{n_{k+1}}\right) \text {. }
$$

Next, using (2.4), we majorize the latter by

$$
\text { Const } \exp \left(-k^{2}+k \log \xi-k \frac{1}{2} Q \sqrt{\sigma}\right) \leq \exp \left(-(k+1)^{2}\right)
$$

if we assumed $Q>\frac{\log \xi+2}{\sqrt{\sigma}}$

Thus the inductive step is done and (2.3) is proved.

Step 4. Diameters of intermediate $U_{n}$ 's. Fix arbitrary $N_{k} \leq n \leq N_{k+1}, k \geq 2$. Denote $r=\operatorname{diam} U_{N_{k}}$. For $z$ which is the origin of $V_{k}=B\left(z, \xi^{k+1} r\right)$ (see Step 1) we have by the definitions

$$
B\left(z, \xi^{k} r\right) \subset\left(V_{k}\right)_{\left[n-N_{k}\right.} .
$$

We now apply the estimate $\left(1.5^{\prime}\right)$ of Lemma 1.4 to

$$
B^{1}=U_{N_{k}}, \quad B^{2}=B^{\prime}=B\left(z, \xi^{k-1} r\right), \quad B=B\left(z, \xi^{k} r\right)
$$

and obtain

$$
\frac{\operatorname{diam} U_{n}}{\operatorname{diamComp} f^{-\left(n-N_{k}\right)}\left(B\left(z, \xi^{k-1} r\right)\right)} \leq C_{4} 2^{L} \xi^{-(k-1) 2^{-K}} .
$$

So for $C_{5}:=C_{4} 2^{L} \xi^{2^{-K}}$ and $\hat{\xi}:=\xi^{2^{-K}}$ we have

$$
\operatorname{diam} U_{n} \leq C_{5} \hat{\xi}^{-k}
$$

If $\xi$ is large then also $\hat{\xi}$ is large.

(To apply (1.4) we worked hard to know that $U_{N_{k}}$ "maps" by $f^{-n_{k+1}}$ to $U_{N_{k+1}}$ together with a wide collar, with bounded criticality. The estimate (2.3) of diam $U_{N_{k}}$ stronger than (2.4) is an interesting byproduct which will not be used in this paper.) 


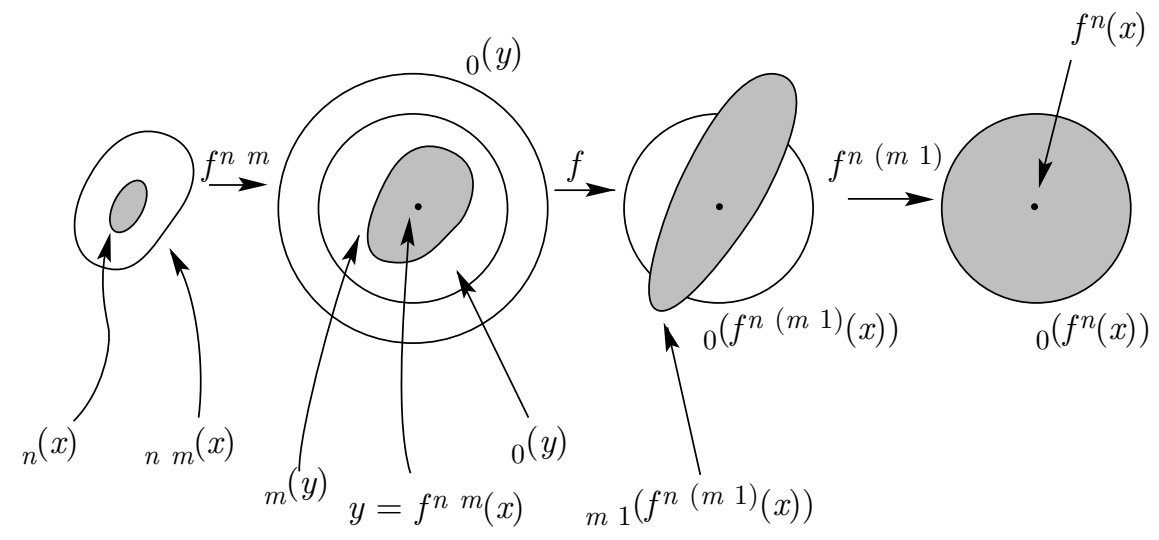

FiguRE 2

Step 5. Conclusion. We have for $N_{k} \leq n \leq N_{k+1}$ (including the case $N_{k+1}=\infty$ ) by $(2.2)$ and $(2.6)$

$$
\frac{\mu\left(U_{n}\right)}{\left(\operatorname{diam} U_{n}\right)^{\alpha^{\prime}}}=\frac{\mu\left(U_{n}\right)}{\left(\operatorname{diam} U_{n}\right)^{\alpha}} \frac{1}{\left(\operatorname{diam} U_{n}\right)^{\alpha^{\prime}-\alpha}} \geq \operatorname{Const}\left(C_{4} 2^{L}\right)^{-\alpha k} \hat{\xi}^{k\left(\alpha^{\prime}-\alpha\right)} \geq \mathrm{Const}
$$

if $\hat{\xi}^{k\left(\alpha^{\prime}-\alpha\right)}>\left(C_{4} 2^{L}\right)^{\alpha}$. For that we should take $Q$ in (0.4) large enough.

Now for every $x \in J(f)$ we produce $U_{n}(x)$ by taking $U_{n} \ni x$ for $U_{0}=B\left(f^{n}(x), \delta\right)$ as above. In (2.7) we can replace each $U_{n}$ by the $\operatorname{disc} B\left(x\right.$, $\left.\operatorname{diam} U_{n}\right)$ which gives (2.1) up to a constant (even larger constant than that in (2.7)). Of course we can get rid of this constant by taking bigger $\alpha^{\prime}$ and considering $r$ small enough.

Observe that

$$
\operatorname{diam} U_{n}(x) \rightarrow 0 \text { as } n \rightarrow \infty
$$

Indeed, if $k(n)$ satisfying $N_{k(n)} \leq n \leq N_{k(n)+1}$ grows to $\infty$ as $n \rightarrow \infty$, then (2.8) holds by (2.6).

Otherwise however, in case there exists $k$ with $N_{k}=\infty$, we obtain $\operatorname{diam} U_{n}(x) \rightarrow$ 0 from Lemma 1.1, part 2.

The only thing we should still check is a part of $(*)$, that there are sufficiently many discs constructed above:

Consider an arbitrary $U_{n}(x)$ with diameter arbitrarily small, less than $\delta$ where $\delta$ is the constant fixed in Step 3. Let $m$ be the least positive integer such that $\operatorname{diam} U_{m}\left(f^{n-m}(x)\right)<\delta$. Denote $f^{n-m}(x)$ by $y$. So $U_{m}(y) \subset U_{0}(y)$, but $\frac{\operatorname{diam} U_{m}(y)}{\operatorname{diam} U_{0}(y)}>$ Const. See Figure 2.

Write $V_{0}(y):=B(y, 2 \delta \xi)$, which is compatible with notation $V_{0}$ from Step 1; we just exhibit the origin.

Apply now from Lemma 1.4 the estimate (1.5), first to $B=\left(V_{0}(y)\right)_{[t}$ with $t=N_{1}=N_{1}(y), B^{\prime}=B(y, \delta \xi), B^{2}=U_{0}(y), B^{1}=U_{m}(y)$ and $f^{-t}$-preimages and 
next $k$ more times, where $k$ is such that $N_{k} \leq n-m \leq N_{k+1}$, similarly to the procedure in Step 2.

We conclude that

$$
\frac{\operatorname{diam} U_{n}(x)}{\operatorname{diam} U_{n-m}(x)} \geq \operatorname{Const}\left(C_{4}^{-1} 2^{-L}\right)^{k} .
$$

Now apply (2.6), which gives that $\operatorname{diam} U_{n-m}(x)<\operatorname{Const} \hat{\xi}^{k}$. We can assume that $\hat{\xi}$ is arbitrarily large, depending on $\beta$. We immediately obtain $\operatorname{diam} U_{n}(x)>$ $\left(\operatorname{diam} U_{n-m}(x)\right)^{\beta}$.

Thus for the disc $D_{n}=B\left(x, \operatorname{diam} U_{n}(x)\right)$ we have found a larger disc $D_{n}^{\prime}=$ $B\left(x, \operatorname{diam} U_{n-m}(x)\right)$ such that both discs satisfy $(2.1)$ and $\left(\operatorname{diam} D^{\prime}\right)^{\beta}<\operatorname{diam} D$. When we continue this procedure we end up with a disc of radius at least $\delta$. This proves $(*)$ and finishes Proof of Theorem A in the absence of parabolic periodic points.

Step 6. Parabolic points. Every critical point out of $J$ has its forward orbit either separated from $J(f)$ or converging to the set $P$ of parabolic points. Now proceeding as in the no parabolic points situation we see there are no problems with (2.2) and (2.6) if $U_{0}$ is far from $P$. There are only problems with proving (2.1) for sufficiently many $r$ 's. However for every $x \in J(f) \backslash \bigcup_{n=0}^{\infty} f^{-n}(P)$ there exists a sequence $n_{j} \rightarrow \infty$ such that $f^{n_{j}}(x)$ is far from $P$. So $(2.1)$ holds for a sequence of discs $B\left(x, r_{j}\right)$ with $r_{j} \rightarrow 0$. This is sufficient for Hausdorff dimension. We conclude $\operatorname{HD}\left(J(f) \backslash \bigcup_{n=0}^{\infty} f^{-n}(P)\right) \leq \alpha$. But $\bigcup_{n=0}^{\infty} f^{-n}(P)$ is countable so of Hausdorff dimension 0 . Hence $\operatorname{HD}(J(f)) \leq \alpha$. Proof of Theorem A is finished.

Remark 2.5. In presence of parabolic periodic points in $J$ I can prove $\alpha \geq \overline{\operatorname{Cap}}(J)$ in Theorem $\mathrm{A}$ under the additional assumption that $\alpha \leq 1$.

In Step 5 to obtain a lot of discs satisfying (2.1), if $z=f^{n}(x)$ hits close to a parabolic point $p$, one uses the estimate $\mu(B(z, r)) \geq$ Const $r^{\alpha}$ obtainable for every $r>\operatorname{dist}(f(z), z)$ by hand, by summing up the respective inequalities for $B_{j}=B\left(f^{j}(z), \operatorname{dist}\left(f^{j-1}(z), f^{j}(z)\right)\right)$ for adequate $j$ 's (including negative ones and considering right branches of $\left.f^{-1}\right)$. The latter inequalities are true by bounded distortion when obtaining $B_{j}$ from large scale sets by backward iteration of $f$. See [DU2].

\section{ERGODICITY}

We shall prove Theorem B here. Let us start with Lemma 3.1 whose assertion is similar to one concerning non-renormalizable quadratic polynomials [Prado] and to a corresponding fact for maps of the interval, see for example [CEbook, Th.2.5.2.3.].

Denote the set of parabolic periodic points by $P=P(f)$.

Lemma 3.1. Let $f: \overline{\mathbb{C}} \rightarrow \overline{\mathbb{C}}$ be a rational map satisfying $\left(0.4^{\prime}\right)$. Then for every $\alpha$ conformal measure $\mu$ on $J(f)$ and $\varepsilon>0$, for $A_{\varepsilon}:=\left\{x \in J(f): \operatorname{dist}\left(f^{n}(x)\right.\right.$, Crit $(f) \cup$ $P(f)) \geq \varepsilon$ for every $n=0,1, \ldots\}, \mu\left(A_{\varepsilon}\right)=0$.

Proof. Consider an arbitrary $x \in A_{\varepsilon}, y=f^{m}(x)$, small $r>0$, and for $y(j):=$ $f^{m-j}(x), j=0,1, \ldots, m$, consider the sequence of components

$$
B_{j}=\operatorname{Comp}_{y(j)} f^{-j}(B(y, r)) \text {, }
$$


i.e. the components such that $y(j) \in B_{j}, j=1,2, \ldots, m$. We prove first that $B_{j} \cap \operatorname{Crit}(f)=\emptyset$ for every $j=1, \ldots, m$. (Compare Mañé's [M], where the assertion is similar, but the assumptions different.)

Observe that we do not need to bother about critical points not in $J$ because their only forward limit points in $J$ can be parabolic periodic points. So the forward orbits of these critical points do not hit $B(y, r)$ for $r$ small enough.

Denote $W=W_{0}=B(y, 10 r)$. If $r$ is small one can find a large $N \leq m$ such that $\left.f^{N}\right|_{\operatorname{Comp}_{y(N)} f^{-N}(W)}$ has no critical points. Let $W_{j}$ denote the component of $f^{-j}\left(W_{[j}\right)$ containing $B_{j}$ respectively. Let $n^{\prime}$ be the first essential critical time with a critical point $c$ for the sequence $W_{j}$ (cf. Section 2, Definition 2.3) with $b_{j}:=0$ for $j<N, b_{j}:=b_{j}^{\prime} / 2 \Sigma_{N}$ for $j \geq N$, where $b_{j}^{\prime}=\sup _{q}\left|\left(f^{j}\right)^{\prime}(f(q))\right|^{-1}$, the supremum taken over all critical points $q \in J$ whose forward orbits do not meet other critical points, and $\Sigma_{N}:=\sum_{j \geq N} b_{j}^{\prime}$.

Denote $n:=n^{\prime}-1$. As $n^{\prime}>N$, we have $b_{n}^{-1}\left|\left(f^{n}\right)^{\prime}(f(c))\right|^{-1} \leq$ Const. Hence using Lemma 1.2 we obtain $\operatorname{dist}\left(f(c), f^{m-n}(x)\right) \leq$ Const $\Sigma_{N} r$. So $\operatorname{dist}\left(f(c), f^{m-n}(x)\right) \leq$ Const $r$, where the latter Const is arbitrarily close to 0 for $N$ large (i.e. for $r$ small). Hence $\operatorname{dist}\left(c, f^{m-n^{\prime}}(x)\right) \leq$ Const $r^{1 / \nu}$. If $r$ was taken so that $r^{1 / \nu}<\varepsilon$ we obtain a contradiction with $\operatorname{dist}\left(f^{m-n^{\prime}}(x)\right.$, Crit $) \geq \varepsilon$.

In particular we obtain $B_{j} \cap$ Crit $=\emptyset$ for every $j=0, \ldots, m$. In fact we obtain more: because, as we proved, there is no essential critical time $\leq m$, the distortion of the respective branch of $f^{-m}$ on $B(y, r)$ is bounded by a constant.

Observe also that by Lemma 1.1 with $K=0$ we have $\operatorname{diam} B_{n} \rightarrow 0$ as $m \geq n \rightarrow$ $\infty$ uniformly independently of $y=f^{n}(x)$.

The result is that $\left.f\right|_{A_{\varepsilon}}$ is expanding. Let us cover $A_{\varepsilon}$ by small discs $D_{1}, \ldots, D_{J} \subset$ $\overline{\mathbb{C}}$ with origins at $x_{1}, \ldots, x_{J}$ respectively, and replace $f$ by its iterate $F:=f^{k}$ so that each branch $G_{j, s}, s=1, \ldots, S_{j}$, of $F^{-1}$ on $D_{j}$ such that $G_{j, s}\left(D_{j}\right)$ intersects $A_{\varepsilon}$ maps the closure of $D_{j}$ into some $D_{i}$. We make a choice: $i=\eta(j, s)$. We consider a 1-sided Markov chain $\mathcal{M}$ of $\sum_{j=1}^{J} S_{j}$ symbols, pairs $(i, s)$, with the 0-1 matrix $M: M_{(i, s),\left(i^{\prime}, s^{\prime}\right)}=1$ iff $i=\eta\left(i^{\prime}, s^{\prime}\right)$. Denote the shift to the left on $\mathcal{M}$ by $\sigma$. In a standard way $\mathcal{M}$ projects to an $F$-invariant repeller $A$ containing $A_{\varepsilon}$, namely $\pi\left(\left(i_{n}, s_{n}\right)_{n=0}^{\infty}\right):=\bigcap_{n=0}^{\infty} G_{j_{0}, s_{0}} \circ \ldots \circ G_{j_{n}, s_{n}}\left(D_{j_{n}}\right)$. Observe that this projection $\pi$ is finite-to-one. (Compare [PUZ, Sec.3, Remark 9].)

We can assume $\mathcal{M}$ is mixing; i.e. an iterate of $M$ has all entries positive. (Otherwise we consider $\mathcal{M}^{\prime}:=\bigcap_{j \geq 0} \sigma^{j}(\mathcal{M})$; hence $\sigma$ maps $\mathcal{M}^{\prime}$ onto itself. Next decompose $\mathcal{M}^{\prime}$ into a finite union of topological Markov chains $\mathcal{M}_{j}$ mixing for an iterate of $\sigma$. $\mu\left(\pi\left(\mathcal{M}_{j}\right)\right)=0$ for each $j$ implies $\mu(\pi(\mathcal{M}))=0$.)

Take a point $y \in A_{\varepsilon} \cap D_{1}$ and its backward trajectory $\gamma$ in $J \backslash$ Crit containing a point having distance to Crit $\cap J$ much smaller than $\varepsilon$, in particular the point not in $A$, next $\gamma$ converging to $A_{\varepsilon}$. This allows a construction of an expanding repeller $A^{\prime}$ strictly containing $A$. We just take new branches of $F^{-1}$, namely $G_{1, S_{1}+1}$ on $D_{1}$, next $G_{J+1,1}$ on $D_{J+1}:=G_{1, S_{1}+1}\left(D_{1}\right)$, next $G_{J+2,1}$ on $D_{J+2}:=G_{J+1,1}\left(D_{J+1}\right)$ etc. along $\gamma$ until $G_{J+n, 1} \circ \ldots \circ G_{J+1,1} \circ G_{1, S_{1}+1}\left(D_{1}\right)$ is back in some $D_{j}, j \leq J$. If $D_{1}$ were taken small enough no critical points obstruct taking these branches. We obtain an extension of $\mathcal{M}$ to a mixing topological Markov chain $\mathcal{M}^{\prime}$. Define $A^{\prime}:=\pi\left(\mathcal{M}^{\prime}\right)$.

For the set of all sequences $\left(\left(i_{n}, s_{n}\right)_{n=0}^{k}\right)$ such that $M_{\left(i_{n}, s_{n}\right),\left(i_{n+1}, s_{n+1}\right)}=1, n=$ $0, \ldots, k-1$, we use the symbol $\mathcal{M}(k)$. We use the analogous notation $\mathcal{M}^{\prime}(k)$ for $\mathcal{M}^{\prime}$. 
So for every $n$

$$
\begin{aligned}
\mu\left(A_{\varepsilon}\right) & \leq \text { Const } \sum_{\left(j_{0}, s_{0}\right), \ldots,\left(j_{n}, s_{n}\right) \in \mathcal{M}(n)} \mid\left(F^{n}\right)^{\prime}\left(\left.G_{\left(j_{0}, s_{0}\right)} \circ \ldots \circ G_{\left(j_{n}, s_{n}\right)}\left(x_{j_{n}}\right)\right|^{-\alpha}\right. \\
& \leq \text { Const } \lambda^{n} \sum_{\left(j_{0}, s_{0}\right), \ldots,\left(j_{n}, s_{n}\right) \in \mathcal{M}^{\prime}(n)} \mid\left(F^{n}\right)^{\prime}\left(\left.G_{\left(j_{0}, s_{0}\right)} \circ \ldots \circ G_{\left(j_{n}, s_{n}\right)}\left(x_{j_{n}}\right)\right|^{-\alpha}\right. \\
& \leq \operatorname{Const} \lambda^{n} \mu\left(A^{\prime}\right)
\end{aligned}
$$

for a number $\lambda<1$. Hence $\mu\left(A_{\varepsilon}\right)=0$.

Proof of Theorem B. Our plan is to find for $\mu$-a.e. $x \in J$ a large family of "boundedly distorted" topological discs $B_{x, \Delta}$, with origins at $x$, diameters tending to 0 so that iterates of $f$ map them with bounded distortion to geometric discs with origins at critical points.

Consider first an arbitrary $x \in J$ such that $\omega(x) \cap \operatorname{Crit}(f) \neq \emptyset$ and $x \notin$ $\bigcup_{n \geq 0} f^{-n}$ (Crit).

Let for every $t \geq 0 c_{t}$ denote a critical point closest to $f^{t}(x)$ and

$$
\Delta_{t}:=\operatorname{dist}\left(f^{t+t\left(c_{t}\right)+1}(x), f^{t\left(c_{t}\right)+1}\left(c_{t}\right)\right),
$$

where $t(c)$ is the largest non-negative integer so that $f^{t(c)}(c) \in \operatorname{Crit}(f)$ (compare Section 2, Basic construction). Let $t_{s}$ be the recursively defined sequence of all consecutive integers such that $\Delta_{t_{s}}<\Delta_{t}$ for all $t<t_{s}$.

Denote for every $c \in$ Crit $\cap J$ the multiplicity of $f^{t(c)+1}$ at $c$ by $\nu(c)$, and denote

$$
r(c, \Delta):=K(c) \Delta^{1 / \nu(c)},
$$

for $K(c)$ defined by

$$
\operatorname{dist}\left(f^{t(c)+1}(z), f^{t(c)+1}(c)\right)=\left(K(c)^{-1} \operatorname{dist}(z, c)\right)^{\nu(c)}+o\left(\operatorname{dist}(z, c)^{\nu(c)}\right) .
$$

Consider arbitrary $s$ with $\Delta_{t_{s}}$ small and $\Delta_{t_{s}} \leq \Delta<\Delta_{t_{s-1}}$. Denote $c_{t_{s}}$ by $c^{\prime}$.

For $B_{0}:=B\left(f^{t_{s}}(x), 3 r\left(c^{\prime}, \Delta\right)\right), W:=B\left(f^{t_{s}}(x), 10 r\left(c^{\prime}, \Delta\right)\right)$ we roughly repeat the Proof of Lemma 3.1. Let $n^{\prime}$ be the first essential critical time and $c$ the related critical point. If $\Delta_{t_{s-1}}$ is small then $n^{\prime}$ is large. The modification of the construction compared with that in Lemma 3.1 is that when defining $b_{j}$, in place of $b_{j}^{\prime}$ we write $\max \left(b_{j}^{\prime}, b_{j+1}^{\prime}, \ldots, b_{j+\hat{t}+1}^{\prime}\right)$. For $n=n^{\prime}-1$ we obtain

$$
\begin{gathered}
\operatorname{dist}\left(f(c), f^{t_{s}-n}(x)\right) \leq \text { Const } b_{n}^{-1}\left|\left(f^{n}\right)^{\prime}(f(c))\right|^{-1} r\left(c^{\prime}, \Delta\right) \\
\leq \operatorname{Const}\left(b_{n+t\left(c^{\prime}\right)+1}^{\prime}\right)^{-1} \Sigma_{N}\left|\left(f^{n+t\left(c^{\prime}\right)+1}\right)^{\prime}(f(c))\right|^{-1} \Delta<\text { Const } \Delta<\Delta_{t_{s}} .
\end{gathered}
$$

The latter Const can be arbitrarily small as it includes $\Sigma_{N}$. This contradicts the definition of $t_{s}$ (we obtained the distance of an image of $x$ from critical values, in the $\Delta_{t}$ 's sense, smaller than $\Delta_{t_{s-1}}$ before the time $\left.t_{s}+t\left(c^{\prime}\right)+1\right)$.

The conclusion is that there exists no essential critical time $n^{\prime}$.

Now for every $s$ and $\Delta_{t_{s}} \leq \Delta<\Delta_{t_{s-1}}$ we define

$$
B_{x, \Delta}:=f_{x}^{-t_{s}}\left(B\left(c_{t_{s}}, r\left(c_{t_{s}}, \Delta\right)\right)\right),
$$

where $f_{x}^{-t_{s}}$ stands for the branch of $f^{-t_{s}}$ mapping $f^{t_{s}}(x)$ to $x$.

If $x \in \bigcup_{n \geq 0} f^{-n}$ (Crit) then for $t$ the first nonnegative integer such that $f^{t}(x) \in$ Crit, denoting $f^{t}(x)$ by $c$, we define $B_{x, \Delta}:=f_{x}^{-t}(B(c, r(c, \Delta)))$. 
Consider now $X:=\left\{x \in J: \operatorname{cl} \bigcup_{n \geq 0} f^{n}(x) \cap\right.$ Crit $\left.\neq \emptyset\right\}$. Suppose there are pairwise disjoint $f$-invariant sets $A_{1}, A_{2}, \ldots, A_{m} \subset X$ of positive measure $\mu$. The family $B_{x, \Delta}$ is a Besicovitch basis; hence the Besicovitch covering theorem holds for it [Gu, Section 1.1, Remark (4), Section 2.2]. An easy conclusion is that this is a density basis; in particular for $\mu$-a.e. $x \in A_{j}, j=1, \ldots, m$,

$$
\lim _{\Delta \rightarrow 0} \frac{\mu\left(B_{x, \Delta} \cap A_{j}\right)}{\mu\left(B_{x, \Delta}\right)}=1 .
$$

If $m>\sharp($ Crit $\cap J)$ then there exist $\Delta$ arbitrarily close to 0 and $x \in A_{i}, y \in$ $A_{j}, i \neq j$, satisfying (3.1) such that both $B_{x, \Delta}$ and $B_{y, \Delta}$ use the same critical point $c^{\prime}$ in the definition. We obtain for $\Delta$ small enough and $r=r\left(c^{\prime}, \Delta\right)$

$$
\frac{\mu\left(B\left(c^{\prime}, r\right) \cap A_{i}\right)}{\mu\left(B\left(c^{\prime}, r\right)\right)}>1 / 2 \text { and } \frac{\mu\left(B\left(c^{\prime}, r\right) \cap A_{j}\right)}{\mu\left(B\left(c^{\prime}, r\right)\right)}>1 / 2
$$

which is a contradiction because $\mu(X) \leq 1$.

Now consider $x \notin X$ such that $\omega(x) \cap P(f) \neq \emptyset$. We define the sequence $t_{s}$ of times of consecutive closest approaches to $P$ similarly to the Crit case. We take branches $f_{x}^{-t_{s}}$ on $B(p, \Delta)$ and consider $B_{x, \Delta}:=f_{x}^{-t_{s}}(B(p, \Delta))$. By the "flower" dynamics close to $P$, each $f^{t_{s}-1}(x)$ is far from $P$; hence critical points from outside $J$ do not interfere. We prove there is no essential critical time for $\Delta$ small enough similarly to the Crit case (with the use of Lemma 1.1).

We prove as in the Crit case that the number of $f$-invariant pairwise disjoint sets of positive $\mu$ measure in $J \backslash X \backslash \bigcup_{\varepsilon>0} A_{\varepsilon}$ does not exceed the number of periodic parabolic orbits. This together with $\mu\left(\bigcup_{\varepsilon>0} A_{\varepsilon}\right)=0$, see Lemma 3.1, finishes the proof of Theorem B.

Remark 3.2. As a by-product the above considerations imply for every $B(x, r), x \in$ $J \backslash P, r$ small enough, that diamComp $f^{-n}(B(x, r)) \rightarrow 0$ uniformly.

We remarked this already in $\S 2$, see (2.8). Here however our assumption on the growth of $\left|\left(f^{n}\right)^{\prime}(f(c))\right|$ is weaker than (0.4), so we need to be careful. This is the reason for modifying the construction instead of referring to Lemma 1.4 where the exponent $-L$ appeared; here we need the exponent -1 ).

\section{Probability absolutely continuous invariant measures}

Proof of Theorem C. We shall prove (0.7) by induction with respect to $n$, more precisely (0.7) with $\sum_{j=0}^{n-1}$ rather than $\sum_{j=0}^{\infty}$. The constants $C_{1}$ and $C_{2}$ will be defined in the course of the proof, independent of $n$ of course.

For $n=0(0.7)$ is trivial (it holds with $C_{1}=1, C_{2}=0$ ). So assume (0.7) for an arbitrary $k \leq n, n \geq 0$ and prove it for $n+1$. Our plan is to prove it first for $x$ such that the time after which the forward orbit of every critical point may hit a small neighbourhood of $x$ is long.

Recall that $\hat{t}$ denotes the largest nonnegative integer such that $f^{\hat{t}}(\operatorname{Crit}(J) \cap J) \cap$ $\operatorname{Crit}(J) \neq \emptyset$.

Next fix $\delta>0$ smaller than $\frac{1}{3} \delta_{0}$ from Lemma 1.1 for $K=0, \lambda=1 / 2$. We shall specify other properties of $\delta$ later on.

We estimate $\mathcal{L}^{m}(1)(x)$ for an arbitrary $m \leq n+1$. The point $x \in J$ is arbitrary; only at the end shall we specify it according to the plan mentioned above. 
We shall denote $\left|\left(f^{s}\right)^{\prime}(y)\right|^{-1}=\lambda(y, s)$. Write

$$
\mathcal{L}^{m}(1)(x)=\sum_{(y, s)} \lambda(y, s)^{\alpha} \mathcal{L}^{m-s}(1)(y)
$$

where we sum over all pairs $(y, s)$ such that $f^{s}(y)=x$ and one of the following two cases holds:

1. (singular) There exists an essential critical time $0<s^{\prime} \leq s$ for $B=B(x, 3 \delta)$ and for the sequence of compatible components $\operatorname{Comp} f^{-t}\left(B_{[t}\right), t=0,1, \ldots, s^{\prime}$, with $f^{s-s^{\prime}}(y) \in \operatorname{Comp} f^{-s^{\prime}}\left(B_{\left[s^{\prime}\right.}\right)$. Let $s^{\prime}$ denote the first such time and the integer $s$ be defined by $s=\min \left(m, s^{\prime}+\hat{t}\right)$.

Here we use the notation of Definition 2.3. We do not specify $b_{j}$ for most of the proof; only at the end do we set

$$
b_{j}:=\frac{b^{j}}{10(1-b)}
$$

for $b<1$ arbitrarily close to 1 .

2. (regular) $s=m$ and there is no essential critical time for the sequence

$$
\operatorname{Comp} f^{-t}\left(B_{[t}\right), t=1, \ldots, m, \quad \text { with } y \in \operatorname{Comp} f^{-m}\left(B_{[m}\right) .
$$

We call each pair $(y, m)$ as in the case 2 . regular. (This follows Nowicki, van Strien terminology.) In this case there exists a branch $g_{y, m}$ of $f^{-m}$ on $B_{[m}$ such that $g_{y, m}(x)=y$. As the distortion of $g_{y, m}$ on $B(x, \delta)$ is bounded by a constant $C$ (cf. Lemma 1.2) we obtain

$$
\lambda(y, m)^{\alpha} \leq C^{\alpha} \frac{\mu\left(g_{y, m}(B(x, \delta))\right)}{\mu(B(x, \delta))} .
$$

So

$$
\sum_{(y, m) \text { regular }} \lambda(y, m)^{\alpha} \leq C^{\alpha} \mu(B(x, \delta))^{-1} .
$$

We set in (0.7) $C_{1}:=C^{\alpha}\left(\inf _{x \in J} \mu(B(x, \delta))\right)^{-1}$.

Now we pass to a harder part of the proof, estimating

$$
\begin{aligned}
& \lambda(y, s)^{\alpha}\left|\mathcal{L}^{m-s}(1)(y)\right| \\
& \quad \leq \lambda(y, s)^{\alpha}\left(C_{1}+C_{2} \sum_{q \in \operatorname{Crit}(f) \cap J} \sum_{j=0}^{m-s-1} \gamma^{j} \frac{\left|\left(f^{j-\hat{t}}\right)^{\prime}\left(f^{\hat{t}+1}(q)\right)\right|^{\alpha / \nu}}{\operatorname{dist}\left(y, f^{j+1}(q)\right)^{(1-1 / \nu) \alpha}}\right)
\end{aligned}
$$

for each singular (i.e. not regular) $(y, s)$.

Observe that $W:=\operatorname{Comp}_{y}\left(B_{\left[s^{\prime}\right.}\right)$ is small. Indeed for $y^{\prime}:=f^{s-s^{\prime}+1}(y)$

$$
\frac{\operatorname{diam} g_{y^{\prime}, s^{\prime}-1}\left(B_{\left[s^{\prime}\right.}\right)}{\operatorname{diam} B_{\left[s^{\prime}\right.}} \leq \operatorname{Const} \lambda\left(y^{\prime}, s^{\prime}-1\right) b_{s^{\prime}-1}^{-1} \text {. }
$$

Here Const includes the distortion constant $C$ from Lemma 1.2, applied under assumption (ii). This assumption (ii) holds if $\delta$ is small enough because then there is an $\varepsilon>0$ such that $B(x, 3 \delta)$ is disjoint from a periodic orbit having two points at least $\varepsilon$ apart. This is so for each $x \in J$ and obviously this implies the same for $f^{-t}(B(x, 3 \delta))$ for each $t \geq 0$.

Denote $f^{s-s^{\prime}+1}$ by $F$. As $W$ is small it contains only one $F$-critical point, which we denote by $c$. Denote the degree of $F$ at $c$ by $\nu(c)$. 
We have

$$
\lambda\left(y, s-s^{\prime}+1\right)=\left|F^{\prime}(y)\right|^{-1} \leq \operatorname{Constdist}(F(c), F(y))^{1 / \nu(c)-1}
$$

where Const depends on $\left.\mid F^{\prime \prime} c\right) \mid$ in the Riemann metric and depends on $\nu(c)$. So

$$
\begin{aligned}
\lambda(y, s)^{\alpha} & \leq \operatorname{Const}\left(\frac{\lambda\left(F(y), s^{\prime}-1\right)}{(\operatorname{dist}(F(c), F(y)))^{1-1 / \nu(c)}}\right)^{\alpha} \\
& \leq \operatorname{Const} \frac{\left(\lambda\left(F(y), s^{\prime}-1\right)^{1-1 / \nu(c)} \lambda\left(F(y), s^{\prime}-1\right)^{1 / \nu(c)}\right)^{\alpha}}{(\operatorname{dist}(F(c), F(y)))^{(1-1 / \nu(c)) \alpha}} \\
& \leq \text { Const } \frac{b_{s^{\prime}-1}^{-\alpha} \lambda\left(F(c), s^{\prime}-1\right)^{\alpha / \nu(c)}}{\left(\operatorname{dist}\left(f^{s}(c), f^{s}(y)\right)\right)^{(1-1 / \nu(c)) \alpha}}
\end{aligned}
$$

After replacing $\nu(c)$ by $\nu(4.4)$ gives the estimate of the $\lambda(y, s)^{\alpha} C_{1}$ summand of $(4.2)$.

Now we shall consider an arbitrary summand

$$
\frac{\lambda(y, s)^{\alpha} C_{2} \gamma^{j} \lambda\left(f^{\hat{t}+1}(q), j-\hat{t}\right)^{\alpha / \nu}}{\operatorname{dist}\left(y, f^{j+1}(q)\right)^{(1-1 / \nu) \alpha}} \text { for } q \in J(f) \cap \operatorname{Crit}(f), 0 \leq j<m-s .
$$

We know that $f^{j+1}(q) \neq c$. Otherwise $f^{j+1+\hat{t}}(q)=c^{\prime}$ is an $f$-critical point, which contradicts the definition that $\hat{t}$ is the largest integer $t$ such that the $f^{t}$-image of a critical point can hit a critical point.

Denote $f^{j+1}(q)$ by $q^{\prime}$. We shall consider 2 cases:

Case I. (i) $\operatorname{dist}\left(q^{\prime}, c\right) \leq 2 \operatorname{dist}(y, c)$ and (ii) $q^{\prime} \in \operatorname{Comp}_{y} f^{-s}\left(B_{\left[s^{\prime}\right.}\right)$ ). (The index $y$ indicates the component containing $y$.)

Observe that by (i) $\frac{\operatorname{dist}\left(F(y), F\left(q^{\prime}\right)\right)}{\operatorname{dist}\left(y, q^{\prime}\right)} \leq$ Const $\left|F^{\prime}(y)\right|$. Hence

$$
\frac{1}{\operatorname{dist}\left(y, q^{\prime}\right)^{1-1 / \nu}}\left|F^{\prime}(y)\right|^{-1} \leq \text { Const } \frac{\left|F^{\prime}(y)\right|^{-1 / \nu}}{\operatorname{dist}\left(F(y), F\left(q^{\prime}\right)\right)^{1-1 / \nu}} .
$$

Hence as in (4.4)

$$
\lambda(y, s)^{\alpha} \frac{\lambda\left(f^{\hat{t}+1}(q), j-\hat{t}\right)^{\alpha / \nu}}{\operatorname{dist}\left(y, f^{j+1}(q)\right)^{(1-1 / \nu) \alpha}} \leq \mathrm{Const} \frac{\lambda\left(f^{\hat{t}+1}(q), s+j-\hat{t}\right)^{\alpha / \nu} b_{s^{\prime}-1}^{-\alpha}}{\operatorname{dist}\left(x, f^{s+j+1}(q)\right)^{(1-1 / \nu) \alpha}} .
$$

Case II. $\operatorname{dist}\left(q^{\prime}, c\right)>2 \operatorname{dist}(y, c)$ or $q^{\prime} \notin \operatorname{Comp}_{y} f^{-s}\left(B_{\left[s^{\prime}\right.}\right)$.

The second condition together with $F(c)=f\left(c^{\prime}\right) \in g_{y^{\prime}, s^{\prime}-1}\left(B_{\left[s^{\prime}\right.}\right)$ implies by Lemma 1.2

$$
\operatorname{dist}\left(F\left(q^{\prime}\right), F(y)\right) \geq \text { Const } b_{s^{\prime}-1}^{2} \operatorname{dist}(F(c), F(y))
$$

hence

$$
\operatorname{dist}\left(q^{\prime}, y\right) \geq \operatorname{Const} b_{s^{\prime}-1}^{2} \operatorname{dist}(c, y)
$$

Of course the first condition also implies this. Using the triangle inequality we obtain also

$$
\operatorname{dist}\left(q^{\prime}, y\right) \geq \frac{1}{2} \operatorname{dist}\left(q^{\prime}, y\right)+\frac{1}{2} \operatorname{Const} b_{s^{\prime}-1}^{2} \operatorname{dist}(c, y) \geq \operatorname{Const} b_{s^{\prime}-1}^{2} \operatorname{dist}\left(q^{\prime}, c\right) .
$$


Denote $w:=\frac{\nu(c)-1}{\nu-1}$. We obtain

$$
\begin{aligned}
& \frac{1}{\operatorname{dist}\left(q^{\prime}, y\right)^{1-1 / \nu}} \leq \operatorname{Const}_{s^{\prime}-1}^{-2(1-1 / \nu)} \frac{1}{\operatorname{dist}(c, y)^{(1-1 / \nu)(1-w)}} \frac{1}{\operatorname{dist}\left(q^{\prime}, c\right)^{(1-1 / \nu) w}} \\
& \quad \leq \operatorname{Const}_{s^{\prime}-1}^{-2(1-1 / \nu)} \operatorname{dist}(F(y), F(c))^{-(1 / \nu(c))(1-1 / \nu)(1-w)} \\
& \quad\left|F^{\prime}\left(q^{\prime}\right)\right|^{-(1 /(\nu(c)-1))(1-1 / \nu) w} \\
& \left.\quad=\text { Const } b_{s^{\prime}-1}^{-2(1-1 / \nu)} \operatorname{dist}(F(y), F(c))\right)^{1 / \nu-1 / \nu(c))} \times\left|F^{\prime}\left(q^{\prime}\right)\right|^{-1 / \nu} \\
& \quad=\text { Const } b_{s^{\prime}-1}^{-2(1-1 / \nu)} A \times B .
\end{aligned}
$$

We have $\left|F^{\prime}(y)\right|^{-1} A \leq$ Const $\frac{1}{\operatorname{dist}(F(c), F(y))^{1-1 / \nu}}($ see $(4.3))$ and $\lambda\left(f^{\hat{t}+1}(q), j-\hat{t}\right)^{1 / \nu} B$ $=\lambda\left(f^{\hat{t}+1}(q), j+1\right)^{1 / \nu}$. Multiplying by $\lambda\left(F(y), s^{\prime}-1\right)$ we obtain finally , as in (4.4), our summand bounded by

$$
\text { Const } \gamma^{j} b_{s^{\prime}-1}^{-2 \alpha(1-1 / \nu)-\alpha} \lambda\left(f^{\hat{t}+1}(q), j+1\right)^{\alpha / \nu} \frac{\lambda\left(F(c), s^{\prime}-1\right)^{\alpha / \nu}}{\operatorname{dist}\left(x, f^{s^{\prime}}\left(c^{\prime}\right)\right)^{(1-1 / \nu) \alpha}} .
$$

Observation 1. Formally each pole $f^{t}(q)$ for the function $\mathcal{L}^{m}(1)$ can occur in the above consideration (case I) for many singular pairs (where $t>s$ ). However it appears in fact at most $\nu$ times. Indeed, let $\left(y_{1}, s_{1}\right),\left(y_{2}, s_{2}\right)$ be two singular pairs for which $f^{t}(q)$ appears, for say $t>s_{1} \geq s_{2}$. Then $s_{1}^{\prime}=s_{1}-\hat{t}, s_{2}^{\prime}=s_{2}-\hat{t}$. Both sets $A:=\operatorname{Comp} f^{-s_{2}^{\prime}}\left(B_{\left[s_{2}^{\prime}\right.}\right)$ and $B:=f^{s_{1}^{\prime}-s_{2}^{\prime}}\left(\operatorname{Comp} f^{-s_{1}^{\prime}}\left(B_{\left[s_{1}^{\prime}\right.}\right)\right)$, where the components contain $f^{\hat{t}}\left(y_{1}\right), f^{\hat{t}}\left(y_{2}\right)$ respectively, contain the point $f^{t-s_{2}^{\prime}}(q)=f^{s_{1}^{\prime}-s_{2}^{\prime}} f^{t-s_{1}^{\prime}}(q)$. Hence $A \supset B$, so $s_{2}^{\prime}$ is the first essential critical time for $\left(y_{1}, s_{1}\right)$; hence $s_{1}=s_{2}$, and $\operatorname{Comp}_{y_{1}} f^{-s_{1}}\left(B_{\left[s_{1}^{\prime}\right.}\right)=\operatorname{Comp}_{y_{2}} f^{-s_{2}}\left(B_{\left[s_{2}^{\prime}\right.}\right)$ contain both $y_{1}, y_{2}$ and $F\left(y_{1}\right)=F\left(y_{2}\right)$

As all pairs for given $t, q$ have the same $s^{\prime}=s_{1}+\hat{t}=s_{2}+\hat{t}$ we can write $s^{\prime}=s^{\prime}(q, t)$.

Observation 2. Given singular $(y, s)$ the pole $f^{s}(c)$ may appear many times, when other poles $f^{t}(q), t>s$, are replaced by it (case II). However for each $t$ there are at most $\sharp(\operatorname{Crit}(f) \cap J)$ such poles. So we obtain the coefficient at $\frac{1}{\left(\operatorname{dist}\left(x, f^{s}(c)\right)\right)^{\alpha(1-1 / \nu)}}$ bounded by

$$
\begin{aligned}
& \text { Const } b_{s^{\prime}-1}^{-2 \alpha(1-1 / \nu)-\alpha} \lambda\left(F(c), s^{\prime}-1\right)^{\alpha / \nu} \sum_{q \in \operatorname{Crit}(f) \cap J, 0 \leq j \leq m-s-1} \gamma^{j} \lambda\left(f^{\hat{t}+1}(q), j+1\right)^{\alpha / \nu} \\
& \leq \text { Const } b_{s^{\prime}-1}^{-2 \alpha(1-1 / \nu)-\alpha} \lambda\left(F(c), s^{\prime}-1\right)^{\alpha / \nu} .
\end{aligned}
$$

This is so because the series converges (exponentially) to a constant.

Conclusion of estimates. Replacing $b_{s^{\prime}-1}^{-2 \alpha(1-1 / \nu)-\alpha}$ by Const $\gamma^{\left(s^{\prime}-1\right) / 2}$ we obtain

$$
\begin{aligned}
& \left|\mathcal{L}^{m}(1)(x)\right| \leq \\
& C_{1}+\operatorname{Const}\left(C_{1}+C_{2}\right) \sum_{q \in \operatorname{Crit}(f) \cap J, 0 \leq t<m} \gamma^{t-s^{\prime}(q, t)} \gamma^{s^{\prime}(q, t) / 2} \frac{\lambda\left(f^{\hat{t}+1}(q), t-\hat{t}-1\right)^{\alpha / \nu}}{\left(\operatorname{dist}\left(x, f^{t+1}(q)\right)^{\alpha(1-1 / \nu)}\right.} .
\end{aligned}
$$

If the minimal $s^{\prime}$ in this sum is large then $\gamma^{s^{\prime} / 2} \ll \gamma^{s^{\prime}}$. So instead of $\gamma^{t-s^{\prime}(q, t)} \gamma^{s^{\prime}(q, t) / 2}$ we can write $\gamma^{t}$ and replace Const $\left(C_{1}+C_{2}\right)$ by a small number $C_{6}$. (We can have just $C_{6}=C_{1}$ and assume in our induction $C_{1}=C_{2}$.) 
The minimal $s^{\prime}$ is large if $x \in B\left(\bigcup_{t=0}^{\hat{t}} f^{-t}\left(\mathrm{Crit}_{1}\right), \eta\right)$, where Crit $1:=\{c \in$ $\operatorname{Crit}(f) \cap J: f^{t}(q) \neq c$ for every $t>0 q \in$ Crit $\}, \quad \eta$ is small enough and $\delta$ is small enough. So we first make the induction step for $x \in B\left(\bigcup_{t=0}^{\hat{t}} f^{-t}\left(\mathrm{Crit}_{1}\right), \eta\right)$.

(Note that we can assume $s^{\prime}$ is so large that Const becomes swallowed but we cannot make $C_{6}$ arbitrarily small without changing $\delta$ in the course of induction. This is so because to guarantee $s^{\prime}$ large we need $\delta$ small, which makes $C_{1}$ large. We can only obtain $C_{6} / C_{1}$ arbitrarily small.)

Next take an arbitrary $x \in J$. If $\delta$ is small enough (this is our last condition on $\delta$ ) then for every singular pair $(y, s)$ we have $\operatorname{diam}\left(\operatorname{Comp}_{y} f^{-s}\left(B_{\left[s^{\prime}\right.}\right)\right)<\eta$; hence $y \in B\left(\bigcup_{t=0}^{\hat{t}} f^{-t}\left(\operatorname{Crit}_{1}\right), \eta\right)$. So the induction hypothesis is satisfied with our $C_{6}$. So we end up with Const $C_{6}$.

Invariant measure. We take $m$ a weak* limit of a subsequence $\mathcal{S}$ of the sequence of measures $\mu_{n}=\frac{1}{n} \sum_{j=0}^{n-1} f_{*}^{j}(\mu)$. Due to this definition $m$ is $f$-invariant. Observe next that for every $n \geq 0$

$$
d f_{*}^{n}(\mu) / d \mu=\mathcal{L}^{n}(1) .
$$

This is so because for every borel $E \subset J$ and $E^{\prime}:=\bigcup_{j=1}^{n} f^{j}(\operatorname{Crit}(f))$

$$
\mu\left(f^{-n}(E)\right)=\sum_{t} \mu\left(f_{t}^{-n}\left(E \backslash E^{\prime}\right)\right)=\sum_{t} \int_{\left.E \backslash E^{\prime}\right)}\left|\left(f_{t}^{-n}\right)^{\prime}\right| d \mu=\int_{E} \mathcal{L}^{n}(1) d \mu .
$$

We use the property that $\mu\left(E^{\prime}\right)=\mu\left(f^{-n}\left(E^{\prime}\right)\right)=0$. This follows from the assumption that $\mu$ is conformal and has no atoms at $f$-critical points. $f_{t}^{-n}$ denote branches of $f^{-n}$. We can divide $E$ into small sets and assume even that $f_{t}^{-n}$ are holomorphic. (If there were an atom at $c \in \operatorname{Crit}(f) \cap J$, then we could not remove $E^{\prime}$ and $\mu(c) \neq\left|f^{\prime}(c)\right|^{-1} \mu(f(c))=0 \cdot \infty$.)

Now as all the functions $\frac{1}{n} \sum_{j=0}^{n-1} \mathcal{L}^{j}(1)$ are uniformly bounded by a $\mu$-integrable function $h$ (the right-hand side expression of $(0.7)$ ) we arrive at $m(E) \leq \int_{E} h d \mu$; hence $m \ll \mu$. (This is standard: one can approximate the indicator function $\chi_{E}$ by continuous functions due to the regularity of our measures and use the weak* convergence of $\mathcal{S}$.)

\section{Appendix. NON-RENORMALIZABle QUADRATIC POLYNOMials. Proof of Theorem D}

Let us recall the terminology [GJ, L]: Let $V^{0} \supset V^{1} \supset \ldots$ be the principal nest of critical puzzle pieces, i.e. pieces containing the critical point 0 . That is,

$V^{k}:=\operatorname{Comp} f^{-q(k)}\left(V^{k-1}\right)$, the component containing 0 , where $q(k)$ is the first time $f^{q(k)}(0) \in V^{k-1}$. Also $\bmod \left(V^{0} \backslash V^{1}\right)>0$.

If $f^{q(k)}(0) \in V^{k}, k \geq 1$, we call $k$ a central return integer; in the opposite case we call it non-central. Denote by $\mathcal{K}$ the set of all non-central return integers and number them $0=k(1)<k(2)<\ldots$. Observe that for every $k(s) \in \mathcal{K}$, for every $k(s)+1 \leq k \leq k(s+1)$, we have $q(k)=q(k(s)+1)$. Denote this integer by $q_{s}$. Finally denote $\bmod \left(V^{k-1} \backslash V^{k}\right)$ by $\bmod _{k}$.

Lemma App1. Consider an arbitrary $x \in \mathbb{C}$. Suppose that $f^{m}(x) \in V^{k}$ and $k^{\prime}:=\sup \left\{l:\right.$ there exists $0 \leq j<m$ such that $\left.f^{j}(x) \in V^{l}\right\} \leq k$. Let $s$ be so that $k(s) \leq k^{\prime}<k(s+1)$. 
Then $f^{m}$ has no critical points in $\mathrm{Comp}_{x} f^{-m}\left(V^{k(s)}\right)\left(\mathrm{Comp}_{x}\right.$ denotes the component containing $x$ ).

Proof. Let $M$ be the largest non-negative integer less than $m$ such that $f^{M}(x) \in$ $V^{k(s)}$ (supposing it exists). Then

$$
f^{M}(x) \notin V^{k(s)+1} .
$$

Indeed suppose that $f^{M}(x) \in V^{t} \backslash V^{t+1}$ where $k(s)+1 \leq t<k(s+1)$. Denote $m^{\prime}=M+q_{s}$. Then $f^{m^{\prime}}(x) \in V^{t-1} \backslash V^{t}$. In particular $f^{m^{\prime}}(x) \notin V^{k}$ because $k \geq t$. Of course for $M<j<m^{\prime}$ we have $f^{j}(x) \notin V^{k}$ as well, because $f^{j}(x) \notin V^{k(s)}$. Hence $m^{\prime}<m$ which contradicts the definition of $M$.

Denote $A_{i}:=\operatorname{Comp}_{f^{i}(x)} f^{-(m-i)}\left(V^{k(s)}\right)$, for each $i=0,1,2, \ldots, m$.

For each $0<j<m-M, A_{m-j}$ contains $f^{m-j}(x) \notin V^{k(s)}$; hence it is disjoint from $V^{k(s)}$ by the Markov property of the puzzle. (By the way this proves Lemma in the case $M$ does not exist.) We show also that

$$
A_{m-M} \subset V^{k(s)} \backslash V^{k(s)+1} \text {. }
$$

Indeed, the disjointness of $A_{m-M}$ from $V^{k(s)+1}$ follows from (App.1) and the fact that both $A_{m-M}$ and $V^{k(s)+1}$ are components of the set returning to $V^{k(s)}$ under the first return map under iteration of $f . A_{m-M} \subset V^{k(s)}$ follows from the Markov structure of the puzzle.

Let $0 \leq m_{1}<m_{2}<\ldots<m_{T}=M<m$ be the sequence of all consecutive integers between 0 and $m$ for which $f^{m_{j}} \in V^{k(s)}$. Suppose that for an integer $j>1$ we have proved already that $A_{m-m_{j}} \subset V^{t_{j}} \backslash V^{t_{j}+1}$ for certain $k(s) \leq t_{j}<k(s+1)$. We have already checked this backward induction hypothesis for $j=T$, i.e. $m_{j}=$ $M$. Then:

If $f^{m_{j-1}}(x) \in V^{k(s)+1}$, we obtain $f^{m_{j}}(x) \in V^{k(s)}$ and $m_{j}-m_{j-1}=q_{s}$. Hence in fact $f^{m_{j-1}}(x) \in V^{t_{j}+1} \backslash V^{t_{j}+2}$ and moreover $A_{m_{j-1}} \subset V^{t_{j}+1} \backslash V^{t_{j}+2}$, with $t_{j}+2 \leq k(s+1)$.

If $f^{m_{j-1}}(x) \in V^{k(s)} \backslash V^{k(s)+1}$ then we use (App.1) and obtain as in (App.2), using the induction hypothesis, that

$$
A_{m_{j-1}} \subset \mathrm{Comp}_{f^{m_{j-1}(x)}} f^{-\left(m_{j}-m_{j-1}\right)}\left(V^{k(s)}\right) \subset V^{k(s)} \backslash V^{k(s)+1} .
$$

Finally as mentioned above $A_{i} \cap V^{k(s)}=\emptyset$ for every $i \neq m_{1}, m_{2}, \ldots, m_{T}, 0 \leq i<$ $m$.

Thus none of $A_{i}, i=0,1, \ldots, m-1$, contains the critical point 0 . This proves our Lemma.

Corollary App2. For each $s=1,2,3, \ldots$ the branch $G_{s+1}$ of $f^{-\left(q_{s+1}-1\right)}$ from $V^{k(s+1)}$ to $f\left(V^{k(s+1)+1}\right)$ extends to $V^{k(s)}$.

Proposition App3. $P:=\mathrm{Comp}_{0} f^{-1} G_{s+1}\left(V^{k(s)}\right) \subset V^{k(s+1)}$. Moreover

$$
\bmod \left(V^{k(s+1)} \backslash P\right) \geq \text { Const } \bmod \left(V^{k(s)-1} \backslash V^{k(s)}\right) .
$$

This proposition is related to (and in fact follows from) [L, Corollary 6] and the considerations preceding it. We shall use this proposition only in the case $k(s)-k(s-1)=1$ so that the modulus on right-hand side in (App.3) is bounded away from 0 ([L, Corollary 6$]$, Note that it can be very small if the cascade of central returns is long, i.e. $k(s)-k(s-1)$ is large). We shall prove however this proposition in the general case because it looks interesting in itself. 
Proof of Proposition App3. Let $0<n<q_{s+1}$ be the first integer for which $f^{n}(0) \in V^{k(s)} \backslash V^{k(s)+1}$. It exists since $q_{s+1}>q_{s}$. By Lemma App.1, $f^{n}(P) \subset$ $V^{k(s)} \backslash V^{k(s)+1}$.

If $f^{n}(P)$ is pre-critical, i.e. the first return to $V^{k(s)-1}$ maps $f^{n}(P)$ into $V^{k(s)}$, then

$$
\bmod \left(V^{k(s)} \backslash f^{n}(P)\right) \geq \bmod _{k(s)} .
$$

If $f^{n}(P)$ is not pre-critical we obtain an even better estimate, cf. [L, Lemma 3].

Now, if $k(s+1)-k(s)=1$ we obtain $\bmod \left(V^{k(j+1)} \backslash P\right) \geq \frac{1}{2} \bmod _{k(s)}$ which proves (App.3).

If $k(s+1)-k(s)>1$, then

$$
\bmod \left(V^{k(s)} \backslash V^{k(s)+2} \backslash f^{n}(P)\right) \geq \text { Const } \min \left(\bmod _{k(s)}, \bmod _{k(s)+2}\right)
$$

By the first mod we mean here the maximal modulus for annuli in $V^{k(s)} \backslash V^{k(s)+2} \backslash$ $f^{n}(P)$ enclosing $f^{n}(P)$. Hence by iterating the right branches of $f^{-q_{s}} \quad k(s+1)-$ $k(s)-2$ times we arrive at

$$
\begin{aligned}
& \bmod \left(V^{k(s+1)-2} \backslash V^{k(s+1)} \backslash f^{n-(k(s+1)-k(s)-2) q_{s}}(P)\right. \\
& \quad \geq \text { Const } \min \left(\bmod _{k(s)}, \bmod _{k(s)+2}\right) .
\end{aligned}
$$

Iterating twice more and taking into account that $\bmod _{k(s)+2}$ is bounded away from 0 we arrive at (App.3).

In the sequel we shall refer to a deep theorem proved by J. Graczyk and G. Świątek [GS1, Theorem C] (for real parameters c) [GS2] and M. Lyubich [L, Theorem I].

Theorem GSL. The moduli $\bmod _{k(s)+1}$ grow at least linearly as $s \rightarrow \infty$.

We shall need in fact only the growth of $\bmod _{k(s)+1}$ to $\infty$. Until now we used only the easy part, that the moduli are bounded away from 0 .

Proof of Theorem D. We construct a sequence of critical puzzle pieces $P^{n}$ by $P^{0}:=$ $V^{k(1)+1}, P^{1}:=\mathrm{Comp}_{0} f^{-1} G_{2}\left(P^{0}\right)$ etc. by induction using each $G_{j}$ once or twice. Here is the precise description:

Let $s=s(n)$ be such that $V^{k(s+1)} \subset P^{n} \subset V^{k(s)}$.

1. If $V^{k(s)+1} \subset P^{n} \subset V^{k(s)}$ and $k(s+1)-k(s)>1$ then define $P^{n+1}:=$ $\mathrm{Comp}_{0} f^{-1} G_{s}\left(P^{n}\right)$.

2. Otherwise define $P^{n+1}:=\operatorname{Comp}_{0} f^{-1} G_{s+1}\left(P^{n}\right)$.

We prove that each $P^{n}$ sits well inside $V^{k(s(n))}$ :

$1^{o}$ If $k(s+1)-k(s)>1$ and $P^{n} \subset V^{k(s)+1}$, then $\bmod \left(V^{k(s)} \backslash P^{n}\right) \geq \bmod _{k(s)+1} \geq$ Const $>0$.

$2^{o}$ If $P^{n} \supset V^{k(s)+1}$ and $k(s)-k(s-1)>1$, then by the construction $V^{k(s-1)+2} \subset$ $P^{n-1} \subset V^{k(s-1)+1}$. Hence

$$
\bmod \left(V^{k(s)} \backslash P^{n}\right) \geq \frac{1}{2} \bmod \left(V^{k(s-1)} \backslash P^{n-1}\right) \geq \bmod _{k(s-1)+1} \geq \text { Const }>0 .
$$

$3^{o}$ If $P^{n} \supset V^{k(s)+1}, k(s)-k(s-1)=1$ and $k(s-1)-k(s-2)>1$, then

$$
\begin{aligned}
\bmod \left(V^{k(s)} \backslash P^{n}\right) & \geq \frac{1}{2} \bmod \left(V^{k(s-1)} \backslash P^{n-1}\right) \geq \frac{1}{4} \bmod \left(V^{k(s-2)} \backslash P^{n-2}\right) \\
& \geq \bmod _{k(s-2)+1} \geq \text { Const }>0 .
\end{aligned}
$$


$4^{o}$ If $P^{n} \supset V^{k(s)+1}$ and $k(s)-k(s-1)=k(s-1)-k(s-2)=1$ then by Proposition App.3 :

$$
\begin{gathered}
\bmod \left(V^{k(s)} \backslash P^{n}\right) \geq \bmod \left(V^{k(s)} \backslash \operatorname{Comp}_{0} f^{-1} G_{s}\left(V^{k(s-1)}\right)\right. \\
\geq \text { Const } \bmod _{k(s-1)} \geq \text { Const }>0
\end{gathered}
$$

In the above cases, in order that they make sense, we assume $n$, hence $s$, are sufficiently large. For small $n$ we have $\bmod \left(V^{k(s)} \backslash P^{n}\right)>0$ by definition and by the first assertion of Proposition App.3.

We conclude that for each $P^{n}$ the respective $g_{n}=G_{s}$ or $G_{s+1}$ extends to an annulus, so that all these annuli have moduli bounded away from 0 . Hence each $g_{n}$ has distortion on $P^{n}$ bounded by a constant independent of $n$.

As in Section 2 (cf. (2.2), in fact much easier) one estimates the $\alpha$-conformal measure versus the diameter:

$$
\frac{\mu\left(P^{n}\right)}{\left(\operatorname{diam} P^{n}\right)^{\alpha}} \geq \xi^{-n} \text { for a constant } \xi>1
$$

One proves this by induction on $n$ loosing at each step a factor bounded by a uniform constant due to the uniformly bounded distortions of $g_{n}$ 's and bounded losses for the $f^{-1}$ 's which follow $g_{n}$ 's ((1.2) in Lemma 1.3).

Consider an arbitrary $x \in J(f) \backslash \bigcup_{j=0}^{\infty} f^{-j}(\{0\})$ containing 0 in its $\omega$-limit set. Let $t_{n}$ be the smallest non-negative integer such that $f^{t_{n}}(x) \in P^{n}$, for each $n=0,1, \ldots$. Then by Lemma App. $1 f^{t_{n}}$ has no critical points in $P^{n}(x):=$ $\operatorname{Comp}_{x} f^{-t_{n}}\left(P^{n}\right)$ and even in $\operatorname{Comp}_{x} f^{-t_{n}}\left(V^{k(s(n))}\right)$. Hence $f^{t_{n}}$ is univalent and has bounded distortion on $P^{n}(x)$. So

$$
\frac{\mu\left(P^{n}(x)\right)}{\left(\operatorname{diam} P^{n}(x)\right)^{\alpha}} \geq \operatorname{Const} \xi^{-n} .
$$

We shall estimate now the diameters of $P^{n}(x)$. Let $\tau_{j}$ be the times of consecutive closest approaches of $f^{\tau}(x)$ to 0 with respect to the sequence $V^{k(s)}, s=1,2, \ldots$. Namely let $\tau_{1}$ be the first time $f^{\tau_{1}}(x) \in V^{k(1)}$. Let $s_{1}$ be the largest integer such that $f^{\tau_{1}}(x) \in V^{k\left(s_{1}\right)}$. By induction, given $\tau_{j}$ such that $f^{\tau_{j}}(x) \in V^{k\left(s_{j}\right)} \backslash V^{k\left(s_{j}+1\right)}$ we let $\tau_{j+1}$ be the first time $f^{\tau_{j+1}}(x) \in V^{k\left(s_{j}+1\right)}$ and $s_{j+1}$ be the largest integer such that $f^{\tau_{j+1}}(x) \in V^{k\left(s_{j+1}\right)}$.

Denote $f^{\tau_{j}}(x)$ by $y_{j}$. As in Proof of Lemma App.1 we obtain for each $j>1$ that

$$
\operatorname{Comp}_{y_{j}} f^{-\left(\tau_{j}-\tau_{j-1}\right)}\left(V^{k\left(s_{j-1}\right)}\right) \subset V^{k\left(s_{j-1}\right)}
$$

Denote $A_{j}:=\operatorname{Comp} f^{-\tau_{j}}\left(V^{k\left(s_{j-1}\right)} \backslash V^{k\left(s_{j}\right)}\right)$, the component enclosing $x$. We obtain a sequence of disjoint annuli $A_{j}$ nested consecutively around $x$ (in fact with a lot of room between them). By Lemma App. $1 f^{\tau_{j}}$ has no critical point in $A_{j}$; hence $\bmod A_{j}=\bmod \left(V^{k\left(s_{j-1}\right)} \backslash V^{k\left(s_{j}\right)}\right)$.

Given $P^{n}$ and $j=j(n)$ such that $s_{j} \leq s(n)<s_{j+1}$ we consider also the annulus $A(n):=\operatorname{Comp} f^{-\tau_{j}}\left(V^{k\left(s_{j}\right)} \backslash P^{n}\right)$. 
We conclude that

$$
\begin{gathered}
\bmod \left(\operatorname{Comp}_{x} f^{-\tau_{1}}\left(V^{k\left(s_{1}\right)}\right) \backslash \operatorname{Comp}_{x} f^{\left.-\tau_{j(n)}\left(P^{n}\right)\right)>\bmod A(n)+\sum_{i=2}^{j(n)} \bmod A_{i}}\right. \\
\geq \sum_{r=k(s(1))+1}^{k(s(n))} \bmod _{r} \geq \operatorname{Const}(s(n))^{2}
\end{gathered}
$$

where the latter inequality follows from Theorem GSL.

So for each $n$

$$
\operatorname{diam}\left(P^{n}(x)\right) \leq \operatorname{Const} \exp \left(-\operatorname{Const} s(n)^{2}\right) \leq \operatorname{Const} \exp \left(-\operatorname{Const}(n / 2)^{2}\right) .
$$

The latter follows from the construction of $P^{n}$ where each $s=s(n)$ repeats at most twice.

If 0 is not in the $\omega$-limit set of $x$ one can pull-back by backward iterations of $f$ large puzzle pieces to $x$ with bounded distortion which gives $\frac{\mu(B)}{(\operatorname{diam} B)^{\alpha}} \geq$ Const $>0$ for arbitrarily small balls $B$ centered at $x$. The diameters of the puzzle pieces go to 0 with the number of generation going to $\infty$ because the branches of the compositions of $f^{-1}$ under consideration form normal families with constant limits.

Finally the set $\bigcup_{j=0}^{\infty} f^{-j}(\{0\})$ is countable so of Hausdorff dimension 0. (In fact one can consider the puzzle pieces $\operatorname{Comp}_{x} f^{-m}\left(P^{n}\right), n=1,2, \ldots$, where $f^{m}(x)=0$. This pulling back does not destroy the estimates for the measure versus diameter and for the diameters of $P^{n}$ 's.)

These ingredients are sufficient to deduce $\operatorname{HD}(J) \leq \alpha$, cf. Section 2. Theorem $\mathrm{D}$ is proved.

\section{REFERENCES}

[BL] M. Bloch and M. Lyubich, Measurable dynamics of S-unimodal maps of the interval, Ann. Sci. Éc. Norm. Sup. (4) 24 (1991), 545-573.

[CE] P. Collet and J.-P. Eckmann, Positive Lyapunov exponents and absolute continuity for maps of the interval, Ergodic Th. and Dyn. Sys. 3 (1983), 13-46. MR 85j:58092

[CEbook] _ Iterated Maps on the Interval as Dynamical Systems, Birkhäuser, Basel-BostonStuttgart, 1980. MR 82j:58078

[DPU] M. Denker, F. Przytycki, and M. Urbański, On the transfer operator for rational functions on the Riemann sphere, Ergodic Th. and Dyn. Sys. 16 (1996), 255-266. MR 97e:58197

[DU1] M. Denker and M. Urbański, On Sullivan's conformal measures for rational maps of the Riemann sphere, Nonlinearity 4 (1991), 365-384. MR 92f:50097

[DU2] — Capacity of parabolic Julia sets, Math. Zeit. 211 (1992), 73-86. MR 93j:30022

[Gu] M. de Guzmán, Differentiation of Integrals in $\mathbb{R}^{n}$, Lecture Notes in Math. vol. 481. SpringerVerlag, Berlin 1975. MR 56:15866

[Guckenheimer] J. Guckenheimer, Sensitive dependence on initial conditions for one-dimensional maps, Comm. Math. Phys. 70 (1979), 133-160. MR 82c:58037

[GJ] J. Guckenheimer and S. Johnson, Distortion of S-unimodal maps, Ann. of Math. 132 (1990), 71-130. MR 91g:58157

[GS1] J. Graczyk and G. Świạtek, Induced expansion for quadratic polynomials, Ann. Sci. Éc. Norm. Sup. (4) 29 (1996), 399-482. CMP 96:11

[GS2] J. Graczyk and G. Świątek, Holomorphic box mappings, Preprint IHES/M/1996/76.

$[\mathrm{H}]$ E. Hille, Analytic Function Theory, Ginn and Company, Boston 1962. MR 34:1490

[K] A. Katok, Lyapunov exponents, entropy and periodic points for diffeomorphisms, Publ. Math. IHES 51 (1980), 137-173. MR 81i:28022

[L] M. Lyubich, Geometry of quadratic polynomials: moduli, rigidity and local connectivity, Preprint SUNY at Stony Brook, IMS 1993/9. 
[LMi] M. Lyubich and J. Milnor, The Fibonacci unimodal map, J. Amer. Math. Soc. 6 (1993), 425-457. MR 93h:58080

[M] R. Mañé, On a theorem of Fatou, Bol. Soc. Bras. Mat. 24 (1993), 1-12. MR 94g:58188

[N] T. Nowicki, Some dynamical properties of S-unimodal maps, Fund. Math. 142 (1993), 4557. MR 94c:58111

[McM] C. McMullen, Complex Dynamics and Renormalization, Ann. of Math. Studies 135, Princeton University Press, 1994. MR 96b:58097

[NS] T. Nowicki and S. van Strien, Invariant measures exist under a summability condition for unimodal maps, Invent. Math. 105 (1991), 123-136. MR 93b:58094

[Prado] E. Prado, Ergodicity of conformal measures for quadratic polynomials, Manuscript, May 23, 1994.

[P1] F. Przytycki, Lyapunov characteristic exponents are non-negative, Proc. Amer. Math. Soc. 119(1) (1993), 309-317. MR 93k:58193

[P2] Invariant measures for iterations of holomorphic maps, In "Linear and Complex Analysis. Problem Book 3" Part II, Eds. V. P. Havin, N. K. Nikolski. Lect. Notes in Math. 1574, Springer (1994), 450-454. MR 96c:00001b

[P3] _ On measure and Hausdorff dimension of Julia sets for holomorphic Collet-Eckmann maps, In "International conference on dynamical systems, Montevideo 1995 - a tribute to Ricardo Mañé", (Eds) F. Ledrappier, J. Lewowicz, S. Newhouse, Pitman Res. Notes in Math. 362, Longman (1996), 167-181.

[PUbook] F. Przytycki and M. Urbański, To appear.

[PUZ] F. Przytycki, M. Urbański, and A. Zdunik, Harmonic, Gibbs and Hausdorff measures on repellers for holomorphic maps, I. Ann. of Math. 130 (1989), 1-40. MR 91i:58115

[R] M. Rees, Positive measure sets of ergodic rational maps, Ann. Sci. Éc. Norm. Sup. (4) 19 (1986), 383-407. MR 88g:58100

[S] D. Sullivan, Conformal dynamical systems, In "Geometric Dynamics", Lec. Notes in Math., vol. 1007, Springer, New York (1983), 725-752. MR 85m:58112

[Shi] M. Shishikura, The Hausdorff dimension of the boundary of the Mandelbrot set and Julia set, Preprint SUNY at Stony Brook, IMS 1991/7.

[U] M. Urbański, Rational functions with no recurrent critical points, Ergodic Th. and Dyn. Sys. 14.2 (1994), 391-414. MR 95g:58191

[Y] J.-Ch. Yoccoz, Talks on several conferences.

Institute of Mathematics, Polish Academy of Sciences, ul. Śniadeckich 800950 Warszawa, Poland

E-mail address: feliksp@impan.impan.gov.pl 